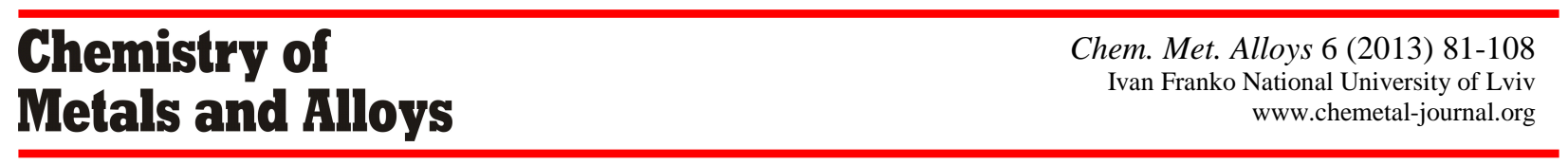

\title{
PAULING FILE verifies / reveals 12 principles in materials science supporting four cornerstones given by Nature
}

\author{
Pierre VILLARS $^{1}$, Shuichi IWATA ${ }^{2}$ \\ ${ }^{1}$ Material Phases Data System (MPDS), Unterschwanden 6, CH-6354 Vitznau, Switzerland \\ ${ }^{2}$ The University of Tokyo, 5-1-5 Kashiwanoha, Kashiwa City, Chiba, 277-8563, Japan \\ * Corresponding author. E-mail: villars.mpds@bluewin.ch
}

Received December 3, 2013; accepted December 25, 2013; available on-line August 30, 2014

Here we outline four cornerstones given by Nature, which on the one hand are responsible for the fact that we are confronted with infinitely many chemical element combinations, on the other hand provide a systematic framework of restraints. To support these four cornerstones, statistical plots and materialsoverview - governing factor maps were used. This led to the establishment of twelve principles, which can be used in materials design as restraints. The principles were verified or derived with the help of the PAULING FILE, the most comprehensive inorganic materials database, consisting of three interconnected parts: structure-diffraction, constitution, and intrinsic physical properties. The principles have general validity and provide a possibility to develop efficient experimentation and calculation exploration strategies.

First cornerstone: Infinitely many chemical element combinations

i) Number of potential chemical element combinations; ii) Number of potential compounds per chemical system and their stoichiometric ratios; iii) Number of potential prototypes per potential compound.

Second cornerstone: Core principle that defines compound formation

1) Compound-formation map principle; 2) Number of chemical elements - atomic-environment type (AET) correlation principle; 3) Active concentration range principle; 4) Stoichiometric ratio condition principle.

Third cornerstone: Core principle that defines ordering of chemical elements within a structure type

5) Simplicity principle; 6) Symmetry principle; 7) Atomic-environment type principle; 8) Chemical element ordering principle.

Fourth cornerstone: Core principle that links the position of chemical elements within a structure type $\leftrightarrow$ in the Periodic System

9) Prototype - Periodic System correlation (chemistry) principle; 10) Structure stability map principle; 11) Generalized AET stability map principle; 12) Complete solid solution stability map principle.

Database / Governing factors / Data mining / Inorganic compounds / Intermetallic compounds / Materials informatics

\section{Introduction}

Confronted with the explosion of computing power, as well as of materials data, Gray proposed in 2009 the Fourth Paradigm of Science: Data-Intensive Discovery through Data Exploration (eScience) [1], which means electronically unify experiment, theory and computation. The Executive Office of the President of the United States, National Science and Technology Council, launched mid-2011 the whitepaper Materials Genome Initiative for Global Competitiveness [2], having as major aim to shorten the time between discovery of advanced materials and their industrial application by at least a factor two. Reflecting these new trends, many ideas have been proposed to explore new dimensions, trying to derive knowledge from a collection of data. To show a clear direction for such trends, we want to draw a roadmap in this work by taking advantage of scientific data, namely in the case of this publication we select scientific data on inorganic compounds (materials).

Three key developments during the past decades have opened up unprecedented opportunities, namely: 1) The power of high-speed computers has reached incredible levels, and their price has decreased. 2) Computational materials science, algorithmic developments, and sophisticated software systems for simulation are advancing at increasing rates. In particular, so-called first-principles methods are now established that can predict the prototype (crystal 
structure) and intrinsic physical properties of inorganic compounds by the only use of fundamental constants. The computed results are often comparable with experimental data. In a similar way, the CALPHAD approach has reached maturity, and is able to calculate phase diagrams that can be compared with experimentally determined phase diagrams. 3) High bandwidth communication at extremely low cost has revolutionized global collaboration and knowledge exchange.

Despite the enormous importance of inorganic compounds for our industrialized society in areas such as housing, energy, transportation, civil engineering, communication, and health, mankind's knowledge of inorganic compounds is astoundingly sparse. For example, only about $16 \%$ of all possible ternary chemical systems have been at least partly characterized. In the case of inorganic compounds containing four or more chemical elements, this fraction drops to $0.6 \%$ or less [3]. In fact, the remarkable accomplishments in the development of advanced technology such as aircraft engines, computer processors, magnetic recording devices, or chemical catalysts, rely on the optimization of physical properties of inorganic compounds.

In this publication we focus on inorganic substances. On the one hand it is worth noticing that the number of experimentally investigated inorganic compounds is very low. But on the other hand the ability to calculate crystallographic data and intrinsic physical properties for inorganic compounds from first-principles methods, and phase diagrams with CALPHAD methods, is rapidly growing. These two facts motivated us to explore new ways to build up a Materials Genome, we call it World Materials, database system consisting of two major inter-linked data parts: The experimentally determined data part, to be used as reference, and the (to be) calculated (using first-principles and CALPHAD mass-calculations) data part.

It has to be highlighted that engineering materials are typically multi-phase materials, which are in addition affected by defects, interfaces, and microstructure. Nevertheless the fundamental basis of all these engineering materials is made from the individual inorganic compounds.

Neither the Fourth Paradigm of Science [1] nor the Materials Genome Initiative [2] can be realized without the integration of restraints obtained by "Materials data exploration searching for principles (governing factors) with the aim to formulate restraints", as shown in this work. The infinite number of potential chemical element combinations forces us to develop approaches that are able to reduce this infinite number to a practicable number of the most probable potential inorganic compounds, to be theoretically and experimentally investigated. In addition it is essential to note that the realization of World Materials requires two pre-conditions: i) The first requirement is the introduction of the distinct phases concept to link different kinds of materials data.

This concept was introduced for the PAULING FILE materials database system [3-5], and is implemented in its derived products [6-9]. A phase is defined by the chemical system and the structural prototype and has been given a unique name by a representative chemical formula and, when relevant, a specification. As the linkage of different groups of data was considered as most important, the PAULING FILE was designed as a phase-oriented database, using a fully relational database system. This was achieved by the creation of a distinct phases table, as well as the required internal links. In practice this means that each chemical system has been evaluated and the distinct phases identified based on available information. Finally every database entry has been linked to such a distinct phase. This is also a requirement for linking materials databases created by different teams.

ii) The second requirement is the existence of a comprehensive, critically evaluated materials database system of experimentally determined singlephase materials data, to be used as reference.

The PAULING FILE was launched 20 years ago and represents meanwhile the sole and therefore world-largest data collection of its kind with over 500 '000 data sets (subdivided into structurediffraction, constitution, intrinsic physical properties) from over 150'000 scientific publications, covering in principle all crystalline inorganic compounds with no $\mathrm{C}-\mathrm{H}$ bonds. It is now becoming feasible to use it as a starting reference. PAULING FILE data have been carefully checked, and fully standardized [10-12].

\section{Exploration of materials data searching for principles (governing factors) with the aim to formulate restraints}

One of the most challenging tasks in materials science is the design of new inorganic compounds with beforehand-defined intrinsic physical and/or chemical properties. In order to reach this objective, two different approaches are, in general, explored [4].

1. The first approach is to simulate the motion of the atoms in the inorganic compounds, as well as their electronic interactions, as close to reality as possible by using quantum-mechanical calculations. In theory there is no input other than the laws of quantum mechanics and the atomic number (AN) of the involved chemical elements. Using these calculations the intrinsic properties of inorganic compounds can be understood from first principles (theoretical and simulation branch of science, representing the Second and Third Paradigm of Science [1]).

2. The second approach is based on a pragmatic level. It is a fact that most of our current knowledge in 
chemistry and materials science has been collected empirically, searching for patterns, rules and principles among experimental results (empirical branch of science, representing the First Paradigm of Science [1]).

For both approaches, to make predictions it is necessary to start from experimental variables such as the selection of the chemical elements to be combined, their concentrations, temperature, and pressure. For the first approach we use, in addition, the laws of quantum mechanics, including the atomic number (AN), whereas for the second approach we use tabulated elemental-property parameters (and/or expressions), such as the atomic number (AN), periodic number $(\mathrm{PN})$, atomic size $\left(\mathrm{SZ}_{\mathrm{a}}(\mathrm{AN}, \mathrm{PN})\right)$ and atomic reactivity $\left(\mathrm{RE}_{\mathrm{a}}(\mathrm{AN}, \mathrm{PN})\right)$ [13].

For both approaches the key-position is the structural prototype of the inorganic compound, which represents our 'window' to view the electronic interactions of the atoms within a specific compound. Presently over 32'000 different prototypes have been experimentally established for inorganic compounds. In other words, Nature realizes more than 32'000 different geometrical arrangements of atoms, only for compounds without $\mathrm{C}-\mathrm{H}$ bonds.

Recently several strong patterns in materialsoverviews - elemental-property parameters maps [13-16], considering thousands of data sets of different chemical systems / inorganic compounds (summarizing the content of thousands of scientific publications), have been published. This proves that the underlying quantum mechanical laws can be parameterized by the use of elemental-property parameters (and/or expressions) of the constituent chemical elements. The search for optimal parameters led to relatively simple maps with well-defined stability domains, giving excellent overviews of experimental data for known inorganic compounds. As a direct consequence the maps give some prediction ability.

Before initiating the PAULING FILE project, one of us reviewed the world literature, focusing on intermetallics and alloys, in context with the topic
"Factors Governing Crystal Structures" [17], and came up with nine principles. The validity of these principles was tested on a fair number of compounds in well-defined groups of experimentally determined data sets and showed accuracy in the range of 90$100 \%$. It was concluded, at that time, that we can rely on predictions based on those nine principles with considerable confidence. Applying the nine principles will both reduce the number of systems (or samples) to be investigated and remarkably increase the success rate of finding new compounds.

Now, 20 years later, having access to five times more experimental facts with a coverage extended from intermetallics and alloys to include other inorganic compounds such as e.g. halides and ceramics, the situation has significantly changed. The PAULING FILE contains structural information for over 140'000 inorganic phases (of which about $50 \%$ contain oxygen), compared to Pearson's Handbook of Crystallographic Data for Intermetallic Phases [18], which covered about 28'000 intermetallics and alloys (including sulfides and selenides).

In this work we sometimes focus on the 1'000 most populous prototypes and their representatives. This covers $70 \%$ of the entries in the 2013/14 edition of Pearson's Crystal Data (PCD-2013/14 [6]), which contains crystal structure data from the PAULING FILE. Tables 1 and 2 give some details about the content of PCD-2013/14, and a brief comparison with the competing product ICSD-2013/14 from FIZ/NIST [19]. By comparing the given numbers, two facts become obvious:

1) PCD-2013/14 [6] contains $60 \%$ more entries that ICSD-2013/14 [19], and therefore covers the world literature more comprehensively.

2) PCD-2013/14 [6] contains 31'883 prototypes, i.e. five times more than ICSD-2013/14 [19] with 6'357 prototypes. The PCD-2013/14 data have been carefully checked [10], consequently standardized with the program STRUCTURE TIDY [12] and the prototypes assigned according to the principles defined in [11].

Table 1 Number of distinct chemical systems, distinct phases, and database entries in PCD-2013/14 [6] and number of database entries in ICSD-2013/14 [19] (grouped into unaries, binaries, ternaries, four or more chemical elements).

\begin{tabular}{l|l|l|l|l}
\hline NUMBER OF & CHEMICAL & DISTINCT & DATABASE & DATABASE \\
CHEMICAL & SYSTEMS & PHASES & ENTRIES & ENTRIES \\
ELEMENTS & PCD-2013/14 & PCD-2013/14 & PCD-2013/14 & ICSD-2013/14 \\
\hline Unaries & 95 & 424 & 2,727 & 1,716 \\
Binaries & $2{ }^{\prime} 553$ & $17^{\prime} 672$ & $48^{\prime} 775$ & $30^{\prime} 968$ \\
Ternaries & $17^{\prime} 131$ & $54^{\prime} 673$ & $95^{\prime} 596$ & $60^{\prime} 640$ \\
$>3$ elements & $33^{\prime} 714$ & $68^{\prime} 919$ & $95^{\prime} 567$ & $56^{\prime} 730$ \\
& & & & \\
\hline Total & $\mathbf{5 3} \mathbf{4 9 3}$ & $\mathbf{1 4 1} \mathbf{\prime}^{\prime} \mathbf{6 8 8}$ & $\mathbf{2 4 2} \mathbf{\prime}^{\prime} \mathbf{6 6 5}$ & $\mathbf{1 5 0} \mathbf{\prime}^{\prime} \mathbf{0 5 4}$ \\
\hline
\end{tabular}


Table 2 Number of database entries in PCD-2013/14 [6] compared with ICSD-2013/14 [19].

\begin{tabular}{|c|c|c|}
\hline & PCD-2013/14 & ICSD-2013/14 \\
\hline Number of prototypes & $31^{\prime} 883$ & 6’357 \\
\hline Number of database entries & $242 ’ 665$ & $150 ’ 054$ \\
\hline - refined atom coordinates & $154^{\prime} 847$ & $122 ’ 300$ \\
\hline - assigned atom coordinates & $72 ’ 088$ & $27\urcorner 754$ \\
\hline - no atom coordinates & $15^{\prime} 730$ & - \\
\hline
\end{tabular}

The main aim of the present work was on the one hand to test the nine principles proposed in [17] (based on about $20 \%$ of the by now available facts) and, if necessary, to modify them, and on the other hand to discover additional principles. Finally we ended up with in total twelve principles, derived either from statistical plots or from materials-overview elemental-property parameters maps. Each of them revealed governing factors, which could be used to formulate restraints.

\section{Nature defines cornerstones providing a marvelously rich, but still very rigid systematic framework of restraints}

Below we outline four fundamental cornerstones defined by Nature. The first cornerstone (3.1.) is responsible for the fact that we are confronted with infinitely many chemical element combinations. The second to fourth cornerstones (3.2.-3.4.) provide a very rigid systematic framework of restraints. Each of the latter is supported by four principles, i.e. in total twelve principles. Since the cornerstones reflect underlying natural laws, they have general validity. For comparison purposes, we will briefly outline the nine principles proposed earlier [17] and, when relevant, their extensions, and describe the newly discovered three principles.

\subsection{First cornerstone: Infinitely many chemical element combinations}

The experimental data in the PAULING FILE as included in SpringerMaterials [9], shows that the materials knowledge rate, by comparing the number of potential chemical element combinations with the number of chemical systems where we have at least partial information, is low. This is shown in Fig. 1 for unary to quinternary systems, and it is clearly seen that we have robust knowledge only for unary and binary systems. For ternary systems we are still at the very beginning and for higher systems we have close to no knowledge. This is surprising as, since 1980, materials scientists are mainly working on ternaries and higher systems. Going to higher-order systems leads to an astronomically large number of potential chemical systems to be considered. Fig. 2a shows that the average number of inorganic phases per binary chemical system is 6.9 , for ternaries about 3.2 and for quinternaries and higher systems about 2 (over 200

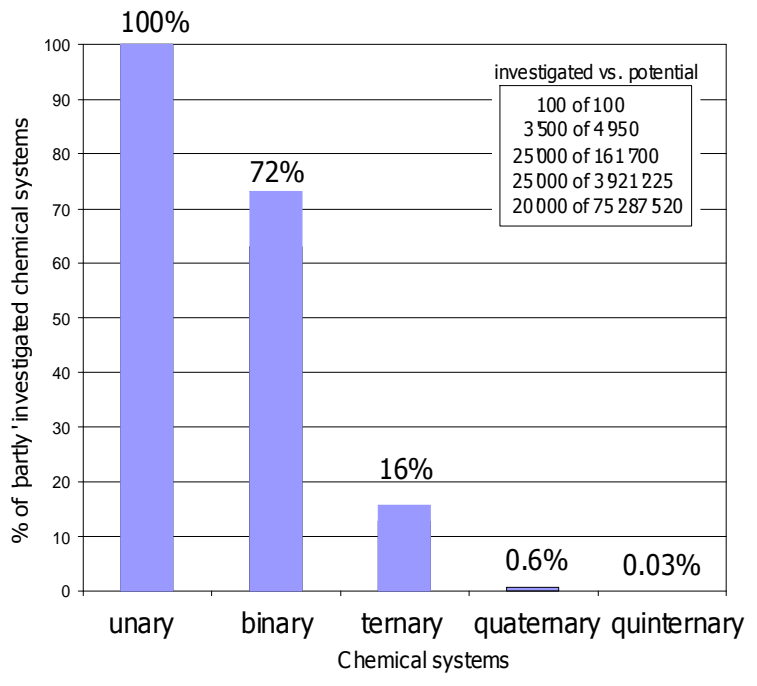

Fig. 1 Percentage of partly or fully investigated chemical systems grouped into unary, binary, ternary, quaternary, and quinternary systems. The inset shows the actual numbers of investigated versus potential systems. The numbers are based on data of SpringerMaterials/PAULING FILE [9].

common stoichiometric ratios occur). In the following we will use the expression basic prototype for a prototype that has no mixed occupancy. Basic prototypes and their representatives are here referred to as daltonide compounds (no significant homogeneity range), which may not always be true. Experimentally investigated systems reveal up to maximum 30 daltonide compounds per system. Fig. 2b shows for 17'083 basic (no mixed site occupancy) ternary compounds 490 different stoichiometric ratios in a ternary concentration triangle, limiting the selection to the 1'000 most populous prototypes and their representatives. Focusing on potential inorganic compounds, going to higher-order systems leads in practice, with an average of about 2 inorganic compounds per system, to an infinitely large variety of potential inorganic compounds to be considered.

Fig. 3 shows that the number of prototypes per literature year has increased approximately linearly from 100 prototypes per year in 1960 to 1'100 prototypes per year in 2010. Nature has so far realized 
P. Villars, S. Iwata, PAULING FILE verifies / reveals 12 principles in materials science ...

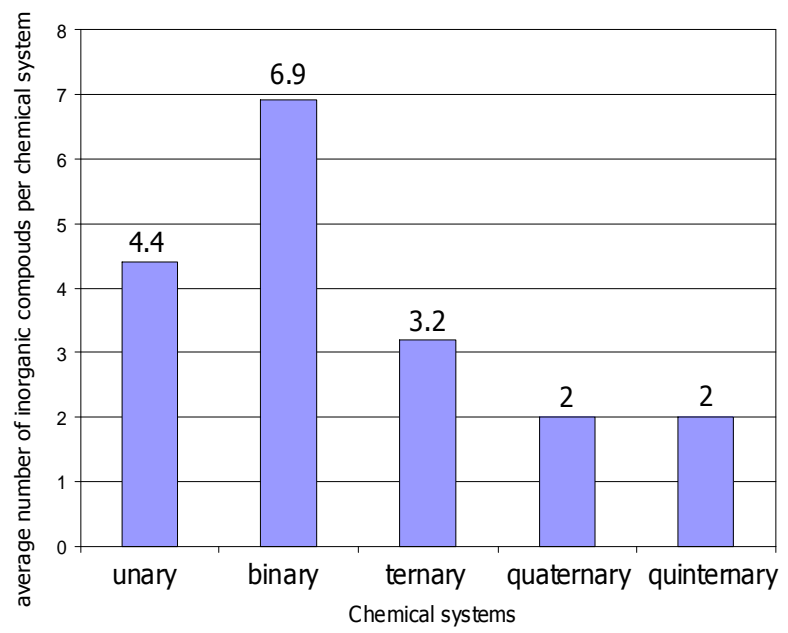

(a)

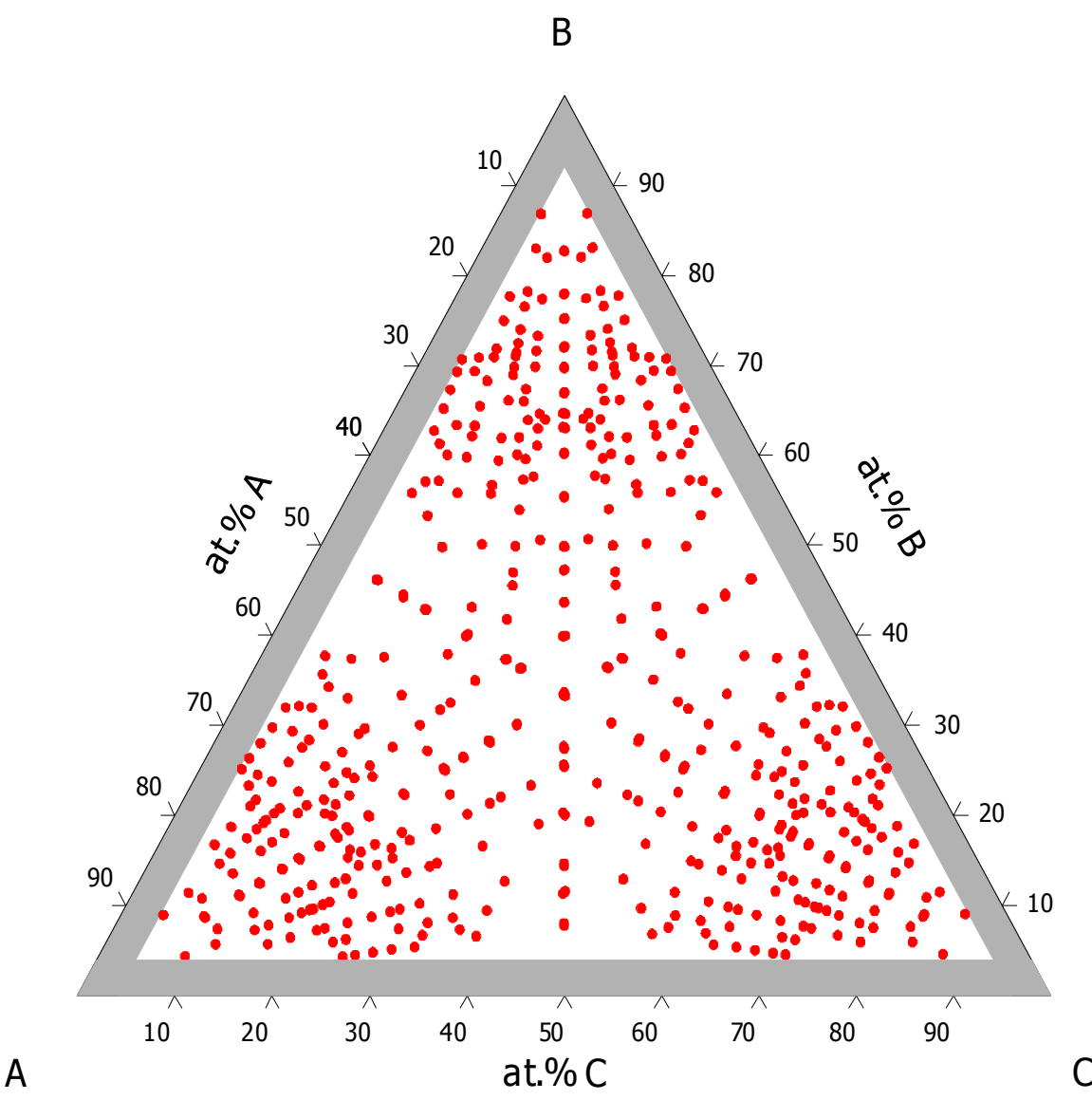

(b)

Fig. 2 (a) Average number of inorganic compounds per chemical system grouped into unary, binary, ternary, quaternary and quinternary systems. The numbers are based on data of SpringerMaterials/PAULING FILE [9]. (b) The over 250 different stoichiometric ratios of the 17'083 daltonide ternary phases shown in a ternary concentration triangle, focusing on the 1'000 most populous prototypes and their representatives in PCD-2013/14 [6]. 


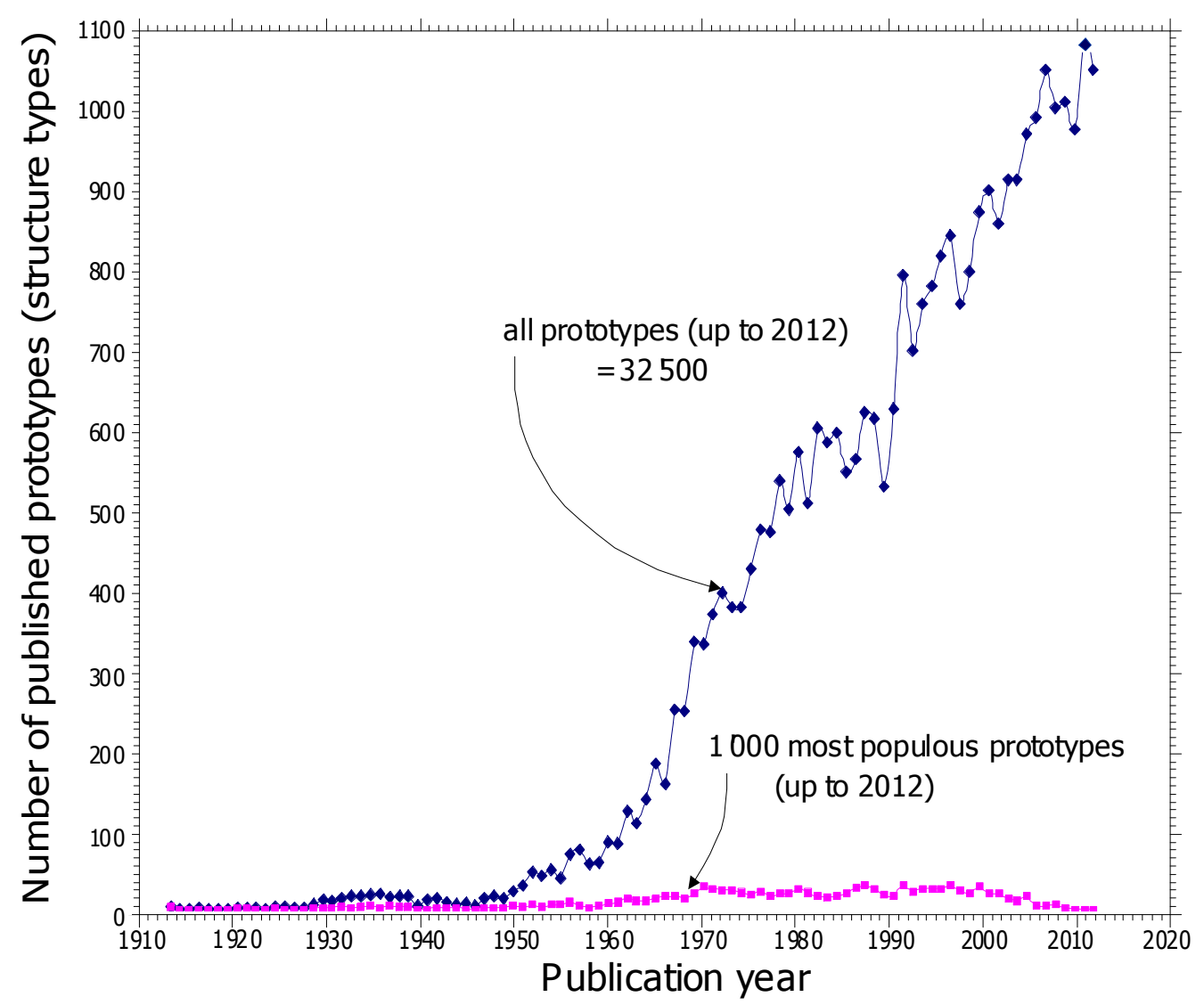

Fig. 3 Number of new prototypes as a function of the publication year. The blue curve considers all prototypes contained in PCD-2013/14 [6], the pink curve considers the 1'000 most populous prototypes in PCD-2013/14 [6].

over 32'000 prototypes for inorganic compounds, and a continued increase would lead to an infinitely large variety of 3-dimensional ways to arrange the atoms within an inorganic compound. Figs. 1-3 support, based on the data of [9], the overall conclusion of the first cornerstone: "Nature provides us with about 100 chemical elements as well as their combinations. A direct consequence of this fact is that there exist an infinite number of chemical element combinations. Furthermore, Nature has worked out a huge number of three-dimensional ways of ordering the chemical elements (atoms) within inorganic compounds. It may be added that the magnetic moments of the chemical elements may be ordered in an even higher number of four-dimensional ways."

This leads, in practice, to a hopeless situation when trying to develop overall-valid efficient experimentation and calculation exploration strategies.

\subsection{Second cornerstone: Laws that define compound formation}

The second cornerstone of Nature sets strict restrictions for the formation of inorganic compounds. The enthalpy of formation has to be negative, otherwise the chemical element combination will not lead to compound. In addition, at constant pressure, Gibbs' phase rule $\mathrm{P}=\mathrm{C}-\mathrm{F}+1$ defines the relation between the number of phases $(\mathrm{P}$, here potential compounds), the number of components $(\mathrm{C}$, here chemical elements) and the degree of freedom $(\mathrm{F})$ of intensive properties such as temperature and composition. It is indirectly possible, with the help of materials overview - elemental-property parameters maps and statistical plots to formulate four principles. Thanks to these four principles it is possible to derive restraints, which can exclude non-former systems (chemical systems where no compounds form), and some pre-conditions to be fulfilled for compound formation.

\section{1) Compound-formation map principle}

Our work from 2008 [13] showed that the periodic number (PN) is by far the most efficient elementalproperty parameter in separating formers (chemical systems where at least one distinct compound exists) from non-formers. PN represents a different enumeration of the chemical elements within the Periodic System (see Fig. 4). In contrast to the atomic number (AN), PN emphasizes the number of valence 


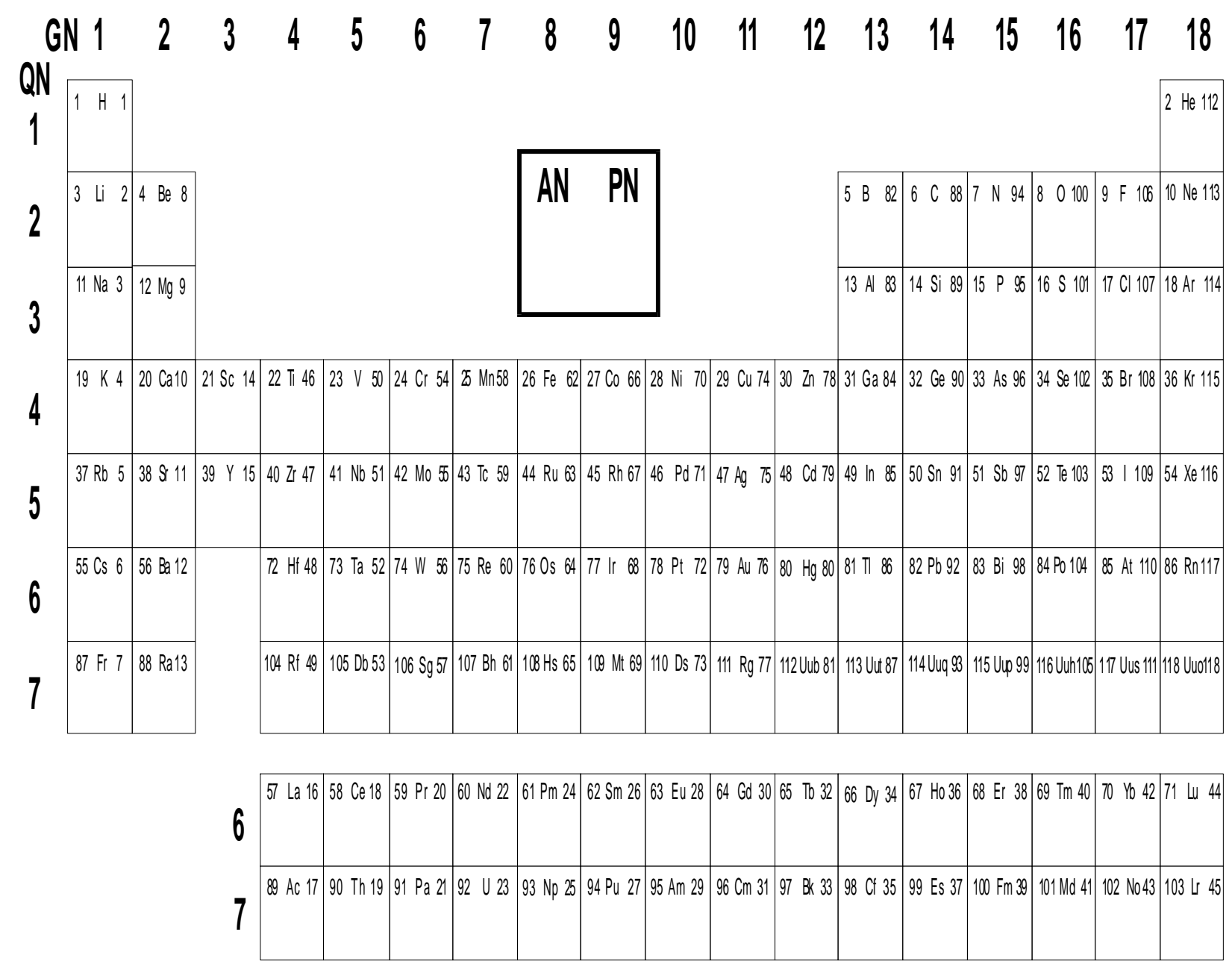

Fig. 4 The four fundamental integer elemental-property parameters: atomic number (AN), periodic number $(\mathrm{PN})$, group number $(\mathrm{GN})$ and quantum number $(\mathrm{QN})$ using Medeleev's periodic system.

electrons, i.e. the periodicity. Fig. 5 shows an idealized two-dimensional compound-formation map for binary $A-B$ systems, using as axes the periodic number of the chemical element $A\left(\mathrm{PN}_{\mathrm{A}}\right)$ versus the periodic number of the chemical element $B\left(\mathrm{PN}_{\mathrm{B}}\right)$. PN as elemental-property parameter works well for binary, ternary, and quaternary systems. However, it was shown in the same publication [13] that the atomic size $\mathrm{SZ}_{\mathrm{a}}$, like the atomic reactivity $\mathrm{RE}_{\mathrm{a}}$, is a direct function of PN and AN strongly reflects the periodicity (valence electrons) of the periodic system. Therefore, $\mathrm{SZ}_{\mathrm{a}}$ and $\mathrm{RE}_{\mathrm{a}}$ can also be used as elementalproperty parameters. Compared with the earlier work of Miedema for binaries [20], and the work in [21], the maps using PN separate the domains more clearly, with an overall accuracy of more than $98 \%$. The advantage of the elemental property parameter PN with respect to parameters such as radius or reactivity is that it is an integer number.

The compound-formation map principle was formulated in 1994 [17] as follows: The size, electrochemical, valence electron, and cohesion- energy factors are the factors governing compound formation. The compound-formation maps predict compound formation in binary [20] and in ternary [21] systems. This principle has been fully verified, and in addition, during the last 20 years, has been significantly improved in its simplicity and accuracy in separating formers from non-formers. Overall one can predict that about $30 \%$ of all chemical element combinations will form no inorganic compound (nonformers), this being true for binary, ternary, and quaternary systems.

\section{2) Number of chemical elements - AET correlation principle}

Fig. 6, a three-dimensional plot, shows the number of point sets with a particular atomic environment (AET) in percent of the total number of point sets for different groups of phases (unary, binary, ternary, quaternary, quinternary, sixnary), limited to the 27 most populous atomic environments and sorted by increasing coordination number $(\mathrm{CN})$. In total close to 120 '000 point sets were considered (all from refined 


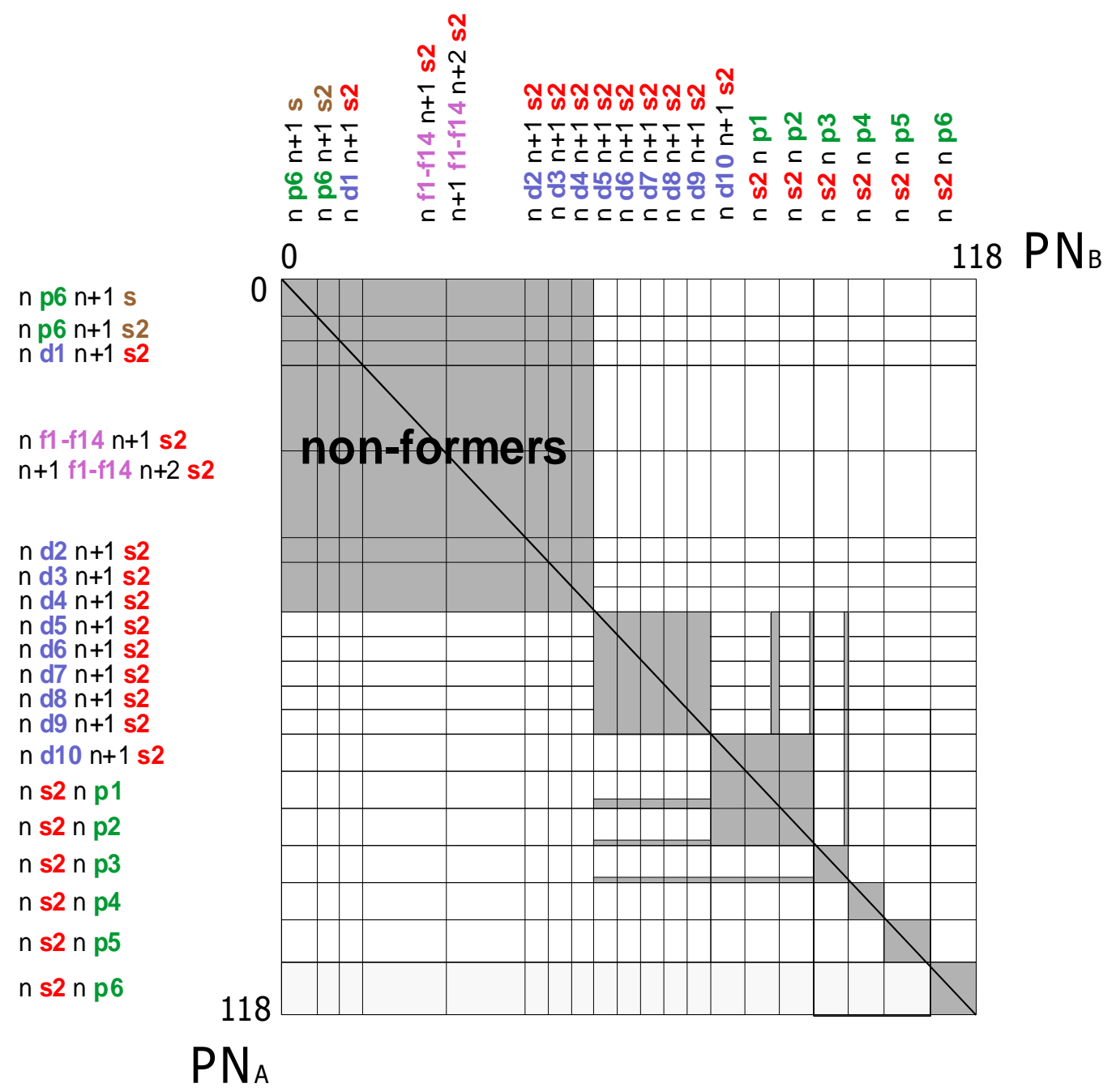

Fig. 5 Two-dimensional compound-formation map for all binary $A-B$ systems using as axes: periodic number of the chemical element $A\left(\mathrm{PN}_{\mathrm{A}}\right)$ versus the periodic number of the chemical element $B\left(\mathrm{PN}_{\mathrm{B}}\right)$, highlighting the correlation to the valence electron configuration of the constituent chemical elements. The non-former areas are indicated with gray color.

structures having no mixed sites) with the following distribution: 238 (one chemical element), 6'856 (two), 17 '083 (three), 4'414 (four), 600 (five), and 165 (six) (see also Table 4). It can be seen that for the chemical elements AETs with $\mathrm{CN}=12$ or higher are strongly preferred. Within binary and ternary compounds the full diversity of AETs is realized, with $\mathrm{CN}=1$ to $\mathrm{CN}=22$. In other words geometrically very different prototypes can be achieved with two or three different chemical elements within inorganic phases. Surprisingly this diversity is reduced when focusing on inorganic phases containing 4-6 chemical elements, and not extended as expected. The plot shows that for quaternary and higher-order inorganic phases AETs with $\mathrm{CN}=6$ or lower are highly preferred. The reason for this artifact can be seen from Fig. 7, where the frequency of the number of point sets (grouped in quaternary, quinternary, sixnary compounds) are plotted versus the nine most populous atomic environment types (AETs) (sorted by increasing coordination numbers $(\mathrm{CN})$ ) and versus the valence electron groups: $s^{1}, s^{2}, p^{1}, p^{2}, p^{3}, p^{4}, p^{5}, d^{1-10}, f^{1-14}$ elements (i.e. columns 1, 2, 13, 14, 15, 16, 17, 3-12 of the Periodic System, lanthanides and actinides). In quaternary and higher-order inorganic compounds $p$ and/or $s$-elements (especially $p^{3}-p^{5}$ elements) occupy the majority of the point-sets. $d^{1-10}$, as well as $f^{1-14}$ elements show an overall low frequency, with the exception of point sets having as AET the tetrahedron (4-a) or the octahedron (6-a) for $d^{1-10}$ elements as central atoms.

The summary of this newly discovered principle: The maximal atomic environment (AET) diversity is reached within binary and ternary inorganic compounds. Unaries (chemical elements) prefer AETs with high coordination number $(\mathrm{CN}=12$ or higher). 


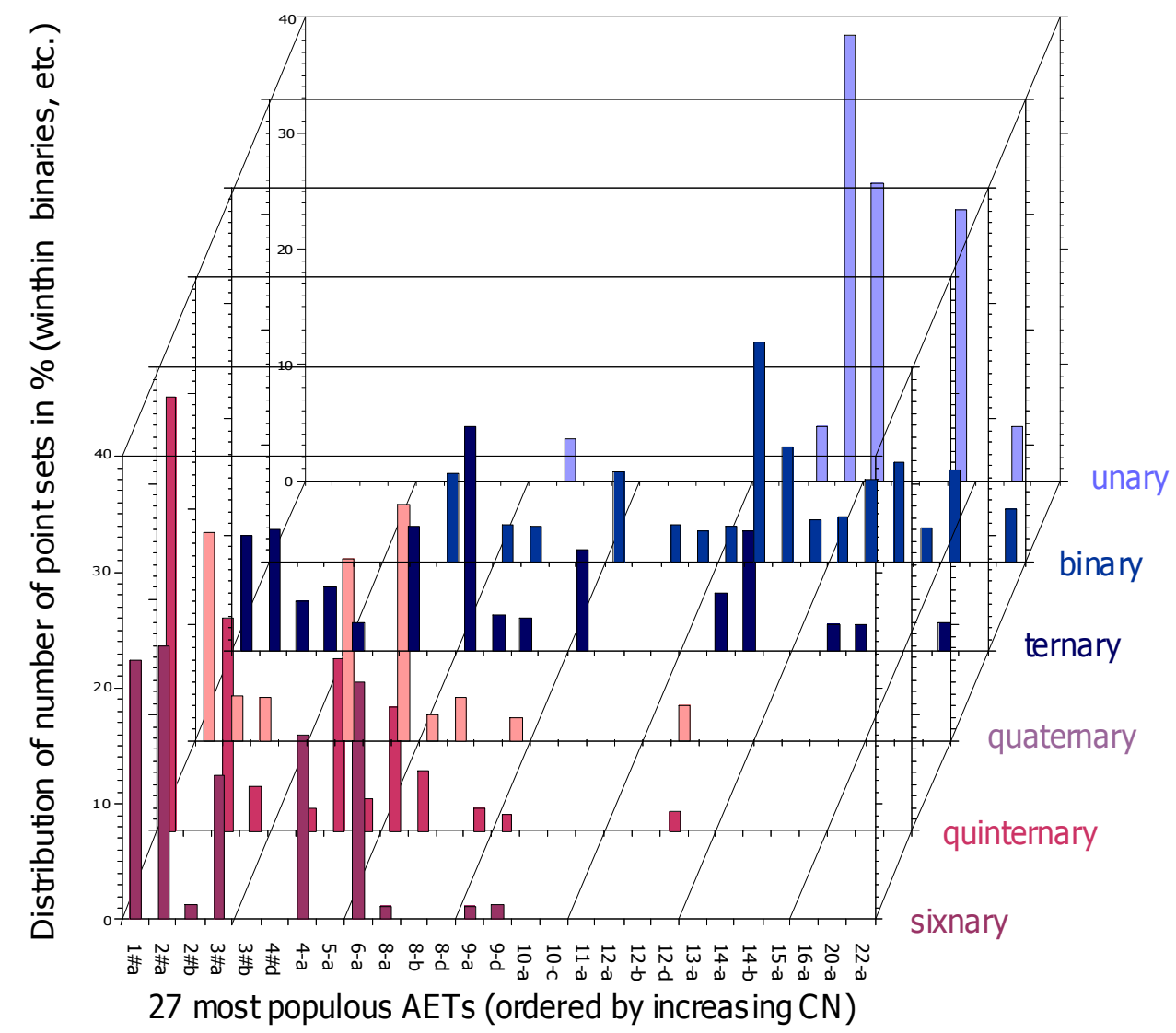

Fig. 6 Frequency of the number of point sets in percent (grouped in unary, binary, ternary, quaternary, quinternary, and sixnary phases) versus the 27 most populous atomic environment types (AETs), sorted by increasing coordination number $(\mathrm{CN})$. The AET codes are explained in Table 3.

Quaternary and higher-order compounds strongly prefer AETs with low coordination number $(\mathrm{CN}=6$ or lower) with the central atoms being mainly $s^{1}, s^{2}$, $p^{1}, p^{2}, p^{3}, p^{4}$ and $p^{5}$ elements. $d^{1}-d^{10}$ and/or $f^{1}-f^{14}$ elements. Multinary compounds predominately have as AET tetrahedra $(\mathrm{CN}=4)$ or/and octahedra $(\mathrm{CN}=6)$.

\section{3) Active concentration range principle}

This principle was formulated in 1994 [17] as follows: Active composition range means that at least 5 at. $\%$ of minority elements is needed to form a compound. This is valid for binary, ternary and quaternary inorganic systems. As a consequence, in binary systems $10 \%$, in ternary systems $15 \%$ and in quaternary systems $38.6 \%$ of the available concentration range (area, volume) is 'inactive' for the formation of daltonide compounds (see gray area in Figs. 8 and 9). This principle has been fully confirmed.

\section{4) Stoichiometric ratio condition principle}

By now $72 \%$ of all binary systems have been investigated and we can state that $95 \%$ of all binary daltonide phases crystallize in one of the following 10 stoichiometric ratios: $A B_{x}$ where $x=6,5,4,3,2,1.67$, $1.5,1.33,1.25$, and 1 (see Fig. 8). For ternaries, 20 years ago it was only possible to list 7 most often occurring stoichiometry ratios. Meanwhile $99 \%$ of the 17 '083 daltonide ternary phases respect the following ternary stoichiometric ratio condition (see Fig. 9): $A B_{x} C_{y}$, where $x$ takes one of the 10 above listed values for the most frequent binary stoichiometric ratios and $y$ is equal to $x$ or an integer divided by $1,2,3$, or 4 , and larger than $x$. In combination with the active composition range principle, this leads to 1 stoichiometric ratio for $A B C(x=y=1)$ compound, 9 possible stoichiometric ratios for $A B_{x} C_{x}(x=y)$ and 819 possible stoichiometric ratios for $A B_{x} C_{y}(x<y)$.

For the stoichiometry $1: 1: 1$ there is only one possibility within a given ternary system $A-B-C$, but for 1:x:y, $x=y$ there are three possible compounds: $A B_{x} C_{x}, A_{x} B C_{x}$, and $A_{x} B_{x} C$, and for 1:x:y, $x \neq y$ six possibilities. This leads in total to 4'636 stoichiometric ratios assuming $A \neq B \neq C$, of which 490 have so far been found experimentally. It is worth mentioning that stoichiometric ratios following certain additional conditions are highly preferred: $A B_{x} C_{y}, x$ equal one of the 10 above listed most frequent values for binary stoichiometric ratios, $y$ equal $x$ or an integer larger than $x$. With these conditions we end up with 769 , compared with the 490 so far experimentally found stoichiometric ratios. 
Table 350 most frequently occurring Atomic Environment Types in PCD-2013/14 [6].

\begin{tabular}{|c|c|c|c|}
\hline $\mathrm{Nr}$ & Counts & AET-CODE & Name \\
\hline 1 & 239814 & $1 \# \mathrm{a}$ & single atom \\
\hline 2 & 188107 & $2 \# \mathrm{a}$ & non-collinear \\
\hline 3 & 143166 & $6-a$ & octahedron \\
\hline 4 & 137309 & 4-a & tetrahedron \\
\hline 5 & 83440 & $3 \# a$ & non-coplanar triangle \\
\hline 6 & 32717 & $12-b$ & cuboctahedron \\
\hline 7 & 24263 & $9-\mathrm{a}$ & tricapped trigonal prism \\
\hline 8 & 21801 & $2 \# \mathrm{~b}$ & collinear \\
\hline 9 & 20129 & $12-\mathrm{a}$ & icosahedron \\
\hline 10 & 14059 & $14-b$ & rhombic dodecahedron \\
\hline 11 & 13684 & $8-\mathrm{a}$ & square prism (cube) \\
\hline 12 & 13548 & $3 \# b$ & coplanar triangle \\
\hline 13 & 12935 & $8-b$ & square antiprism \\
\hline 14 & 12763 & $5-\mathrm{a}$ & square pyramid \\
\hline 15 & 11978 & $5-\mathrm{c}$ & trigonal bipyramid \\
\hline 16 & 8970 & $7-g$ & monocapped trigonal prism \\
\hline 17 & 8270 & $14-\mathrm{a}$ & 14-vertex Frank-Kasper \\
\hline 18 & 7739 & $10-\mathrm{a}$ & fourcapped trigonal prism \\
\hline 19 & 7648 & $6-b$ & trigonal prism \\
\hline 20 & 7507 & $16-\mathrm{a}$ & 16-vertex Frank-Kasper \\
\hline 21 & 7253 & $4 \# \mathrm{c}$ & coplanar square \\
\hline 22 & 6099 & $7-\mathrm{h}$ & pentagonal bipyramid \\
\hline 23 & 5895 & $13-\mathrm{a}$ & pseudo Frank-Kasper (13) \\
\hline 24 & 5865 & $20-\mathrm{a}$ & pseudo Frank-Kasper (20) \\
\hline 25 & 5262 & $12-d$ & anticuboctahedron \\
\hline 26 & 5136 & $4 \# d$ & non-coplanar square \\
\hline 27 & 4388 & $8-d$ & distorted square anti-prism, type a \\
\hline 28 & 3953 & $4 \# \mathrm{~b}$ & tetrahedron, central atom outside \\
\hline 29 & 3515 & $11-\mathrm{a}$ & pentacapped trigonal prism \\
\hline 30 & 3481 & $6-d$ & pentagonal pyramid \\
\hline 31 & 3472 & $15-\mathrm{a}$ & 15-vertex Frank-Kasper \\
\hline 32 & 3286 & $17-d$ & 7-capped pentagonal prism \\
\hline 33 & 3225 & $10-\mathrm{b}$ & bicapped square prism \\
\hline 34 & 3021 & $8-g$ & double anti-trigonal prism \\
\hline 35 & 2731 & $18-\mathrm{a}$ & eight equatorial capped pentagonal prism \\
\hline 36 & 2567 & $11-b$ & pseudo Frank-Kasper (11) \\
\hline 37 & 2469 & $10-\mathrm{c}$ & bicapped square antiprism \\
\hline 38 & 2428 & $8-\mathrm{i}$ & side-bicapped trigonal prism \\
\hline 39 & 2306 & $8-c$ & hexagonal bipyramid \\
\hline 40 & 2086 & $22-\mathrm{a}$ & polarity, eight-equatorial capped hexagonal prism \\
\hline 41 & 2033 & $10-\mathrm{e}$ & distorted equatorial four-capped trigonal prism \\
\hline 42 & 1937 & $5 \# \mathrm{~d}$ & square pyramid, central atom outside of base plane \\
\hline 43 & 1768 & $8-\mathrm{j}$ & distorted square anti-prism, type b \\
\hline 44 & 1735 & $12-f$ & hexagonal prism \\
\hline 45 & 1147 & $14-d$ & bicapped hexagonal prism \\
\hline 46 & 1022 & $7-\mathrm{a}$ & monocapped octahedron \\
\hline 47 & 945 & $18-d$ & sixcapped hexagonal prism \\
\hline 48 & 911 & $20-\mathrm{h}$ & twelve pentagonal faced polyhedron \\
\hline 49 & 910 & $6-\mathrm{h}$ & distorted trigonal prism \\
\hline 50 & 776 & $10-\mathrm{j}$ & polarity bicapped square prism \\
\hline
\end{tabular}


Table 4 Number of chemical elements per prototype (rows) versus number of chemical elements of its representatives (columns) considering the 1'000 most populous prototypes and their representatives in PCD-2013/14 [6].

\begin{tabular}{|c|c|c|c|c|c|c|c|c|c|}
\hline & & \multicolumn{7}{|c|}{ Number of chemical elements per rep resentative } & \multicolumn{2}{c|}{ no. distinct } \\
phases
\end{tabular}

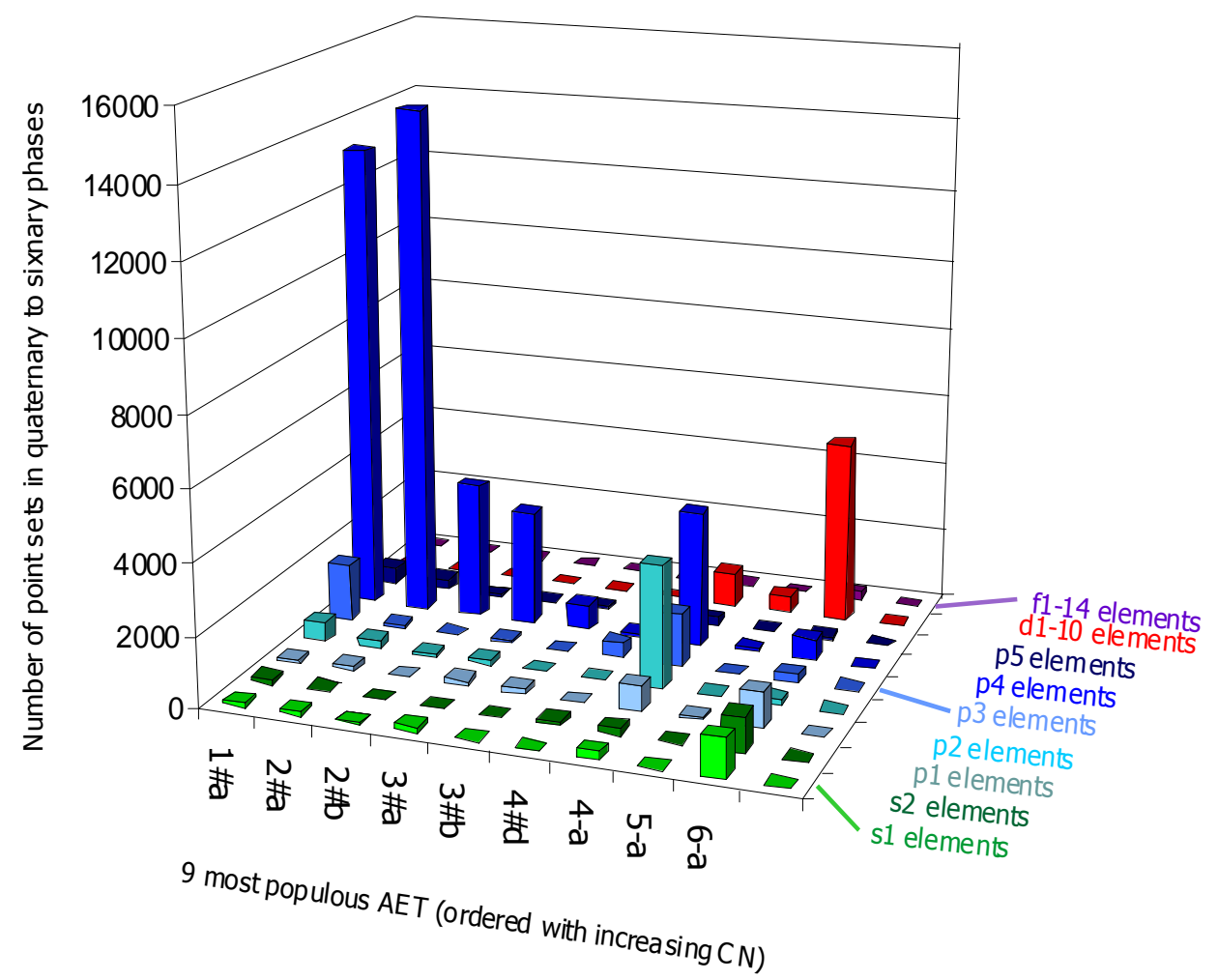

Fig. 7 Number of point sets (grouped in quaternary, quinternary, sixnary phases) versus the 9 most populous atomic environment types (AETs), sorted by increasing coordination number (CN), versus element group: $s^{1}, s^{2}, p^{1}, p^{2}, p^{3}, p^{4}, p^{5}, d^{1-10}, f^{1-14}$-elements. AET: 1\#a, single atom; 2\#a, non-collinear; 2\#b, collinear; 3\#a, non-coplanar triangle; 3\#b, coplanar triangle; 4\#d, non-coplanar square; 4-a, tetrahedron; 5-a, square pyramid; 6-a, octahedron. 


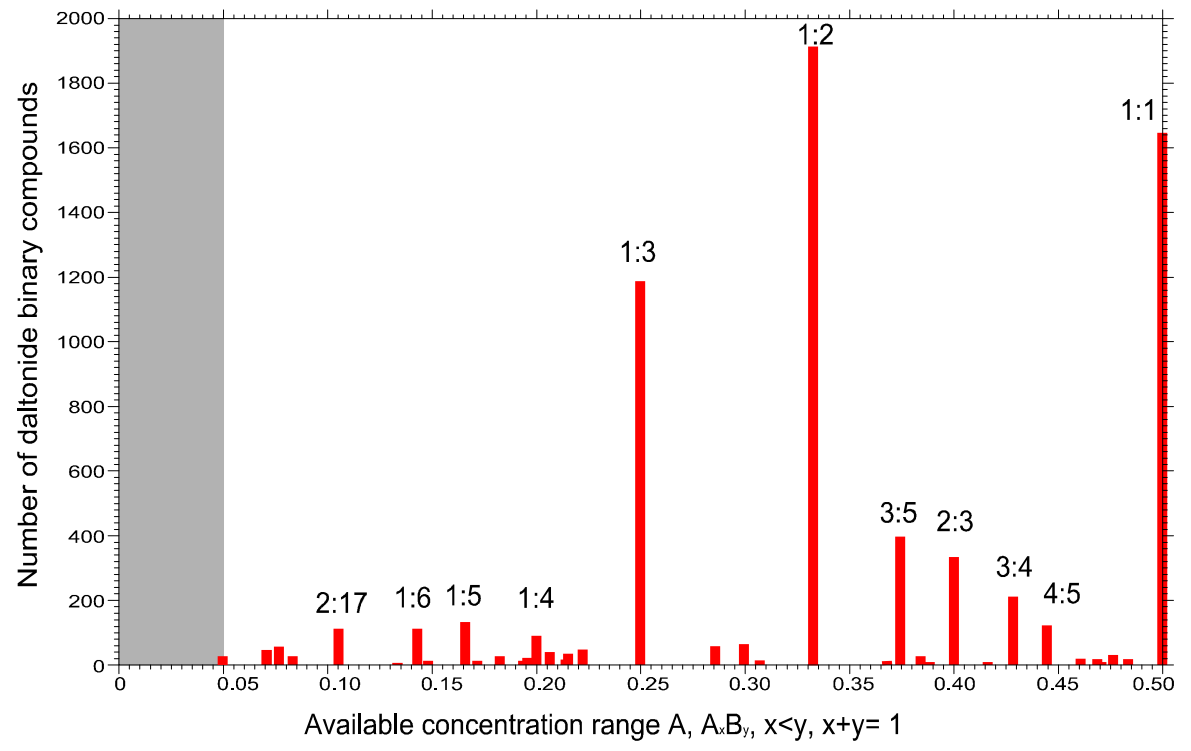

Fig. 8 Number of binary daltonide inorganic phases $A_{x} B_{y}(x<y, x+y=1)$ versus $x$. Numbers indicate frequent $m: n$ ratios $\left(A_{m} B_{n}\right.$ where $m$ and $n$ are integers); the gray color shows the composition range where no binary phases occur.

\section{B}

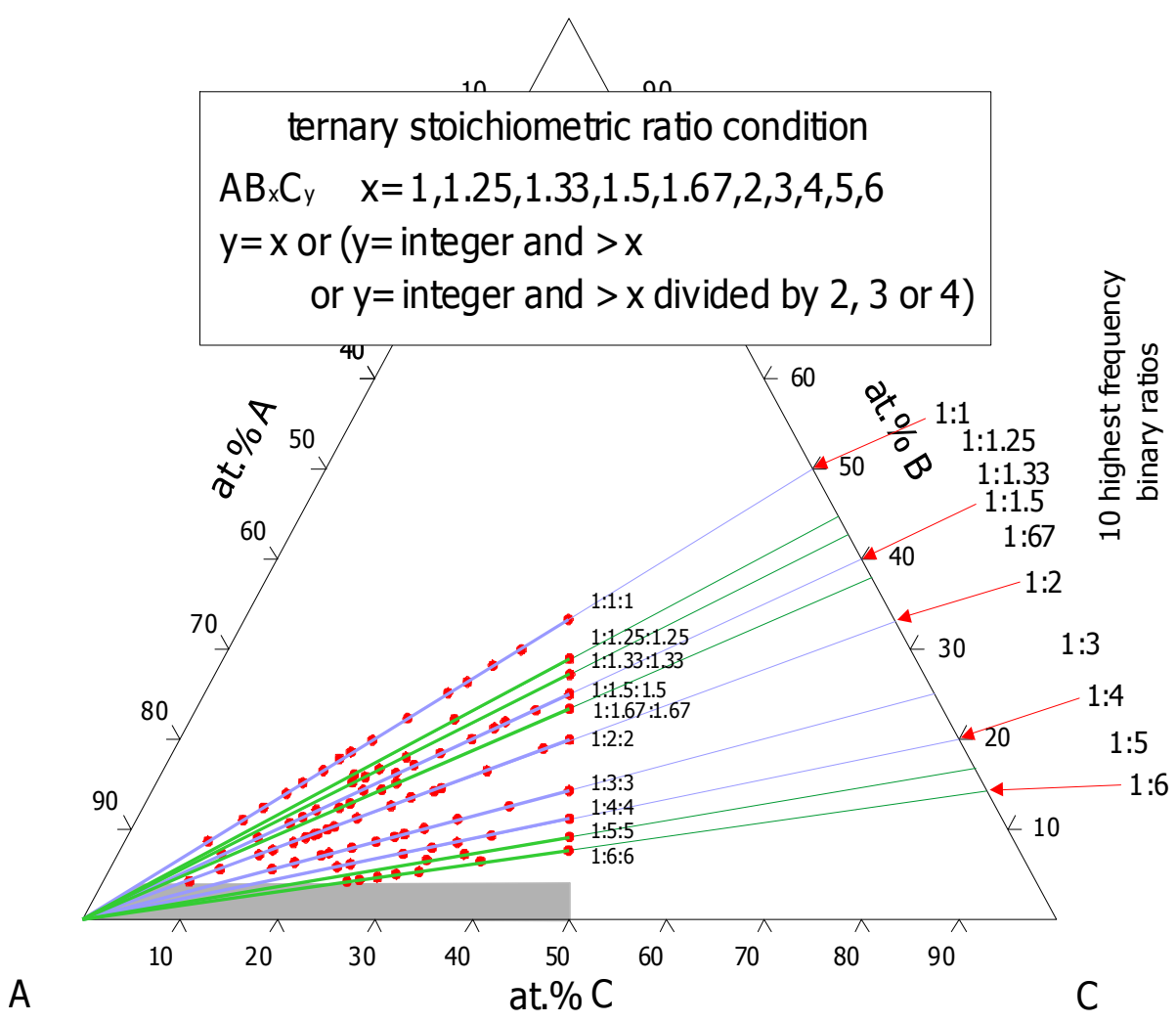

Fig. 9 Occurrence of daltonide inorganic phases in the available concentration range for ternary systems $\left(A_{x} B_{y} C_{z}, x<y<z, x+y+z=1\right)$; the gray area shows where no ternary phases occur. 
The stoichiometric ratio condition principle was formulated in 1994 [17] as follows: The vast majority of daltonide compounds have each point set occupied by just one kind of chemical element. This leads for binaries to the following highly preferred stoichiometric ratios: $1: 1,4: 5,3: 4,2: 3,3: 5,1: 2,1: 3$, $1: 4,1: 5$, and 1:6. This has been fully verified in this work. For ternary intermetallic compounds, the stoichiometric ratios 1:1:1, 1:1:2, 1:1:3, 1:1:4, 1:2:2, $1: 2: 3$, and $1: 2: 4$ predominate. In this work the above given ternary stoichiometric ratio condition was discovered.

The four principles demonstrate with the help of Figs. 6-9, based on data of Pearson's Crystal Data [6] and ASM Alloy Phase Diagram Database [7], that the overall conclusion of the second cornerstone is correct: "Nature provides us with a very rigid systematic framework of restraints, such as laws that define compound formation."

Most efficient in reducing the number of potential chemical element combination is the fact that we can focus on unary to quaternary systems, since with them the full diversity of AETs (as well as prototypes) is achieved. Quinternary and higher-order systems will not lead to inorganic compounds with greater diversity, and therefore not to the discovery of additional principally different geometrical atom arrangements. In other words, all principal prototypes are realized with compounds having four or less chemical elements.

The number of potential chemical element combinations (chemical systems) for unaries to quaternaries is 4'087'975. About $30 \%$ of these systems are non-formers, which leaves about 2'900'000 combinations. Taking the average number of inorganic phases for unaries as 5 , for binaries as 7 , for ternaries as 4 and quaternaries as 2, leads to less than 6'000'000 inorganic phases, preferring one of 10 stoichiometric ratios for binaries, or obeying the stoichiometric ratio condition for ternaries.

Taking into consideration the fact that there exist only four major groups of chemical elements $(s-, \quad p-, d-$ and $f$-elements) supports the above said. From the chemical point of view the quaternaries should represent the maximum of potential diversity that can be achieved. The basic prototypes and their basic representatives among quinternary and higher-order inorganic compounds cannot be built up with chemical elements from five (or more) distinct different chemical element groups, since there exist only four groups, but one or more chemical elements will have to belong to the same group of elements.

\subsection{Third cornerstone: Laws that define ordering} of chemical elements within a structure type

The third cornerstone of Nature sets strict restrictions on the ordering of the chemical elements within a particular inorganic compound. When chemical elements combine to form solids, their crystal structures are beautifully rich, yet systematic patterns underlie this process.

The most striking manifestation of this fact is the existence of so-called prototypes of crystalline inorganic compounds, which can be understood as geometrical templates for large groups of inorganic compounds, e.g. the $\mathrm{NaCl}, \mathrm{cF} 8,225$ prototype has presently 1299 different basic representatives. In other words, different inorganic compounds representing the same prototype are geometrically very similar to each other. There exist, in principle, two main approaches to the classification of crystal structures, one considers as first criterion the overall symmetry (e.g. the Wyckoff sequence [11]), the other one the atomic environments, also called coordination polyhedra, of each site (leads to the atomic environment type AET classification) [22], or of part of the elements [23]. The first classification requires that the published crystallographic data are fully standardized [12], which is consequently done in the PAULING FILE [3-5] and the products derived from it, e.g. Pearson's Crystal Data [6].

The following four principles demonstrate, with the help of four statistical plots and one fundamental crystallographic consideration, the correctness of the third cornerstone.

\section{5) Simplicity principle}

Fig. 10 shows that the large majority of all prototypes of inorganic phases have less than 40 atoms per unit cell, with a maximum of types having around 8 atoms per cell. It is also demonstrated that the majority of all prototypes (and therefore also their representatives) have 3 or fewer atomic environment types (AETs), with a maximum around 2-3 AETs per prototype. The number of point sets per prototype is for the majority lower than 6 , with a maximum around $2-3$ point sets per prototype (see Fig. 11).

This principle was formulated in 1994 [17] as follows: The vast majority of the intermetallic compounds have less than 24 atoms per unit cell. By the inclusion of other inorganic compounds 24 has become 40, nevertheless the average stays at around 10 atoms per unit cell. In addition the vast majority of all crystal structures have three or fewer atomic environment types (AETs) within the crystal structures (single-, two-, and three-environment types). This is still supported. An analog observation can also be made focusing on the number of point sets instead of the number of different AETs.

\section{6) Symmetry principle}

Fig. 12 gives the number of inorganic phases distributed by space group number considering all entries of PCD-2013/14 [6].

The symmetry principle was formulated in 1994 [17] as follows: The vast majority of all intermetallic compounds and alloys crystallize in one of the following 11 space groups: 12, 62, 63, 139, 166, 191, $194,216,221,225$, and 227. By extending to other 
P. Villars, S. Iwata, PAULING FILE verifies / reveals 12 principles in materials science ...

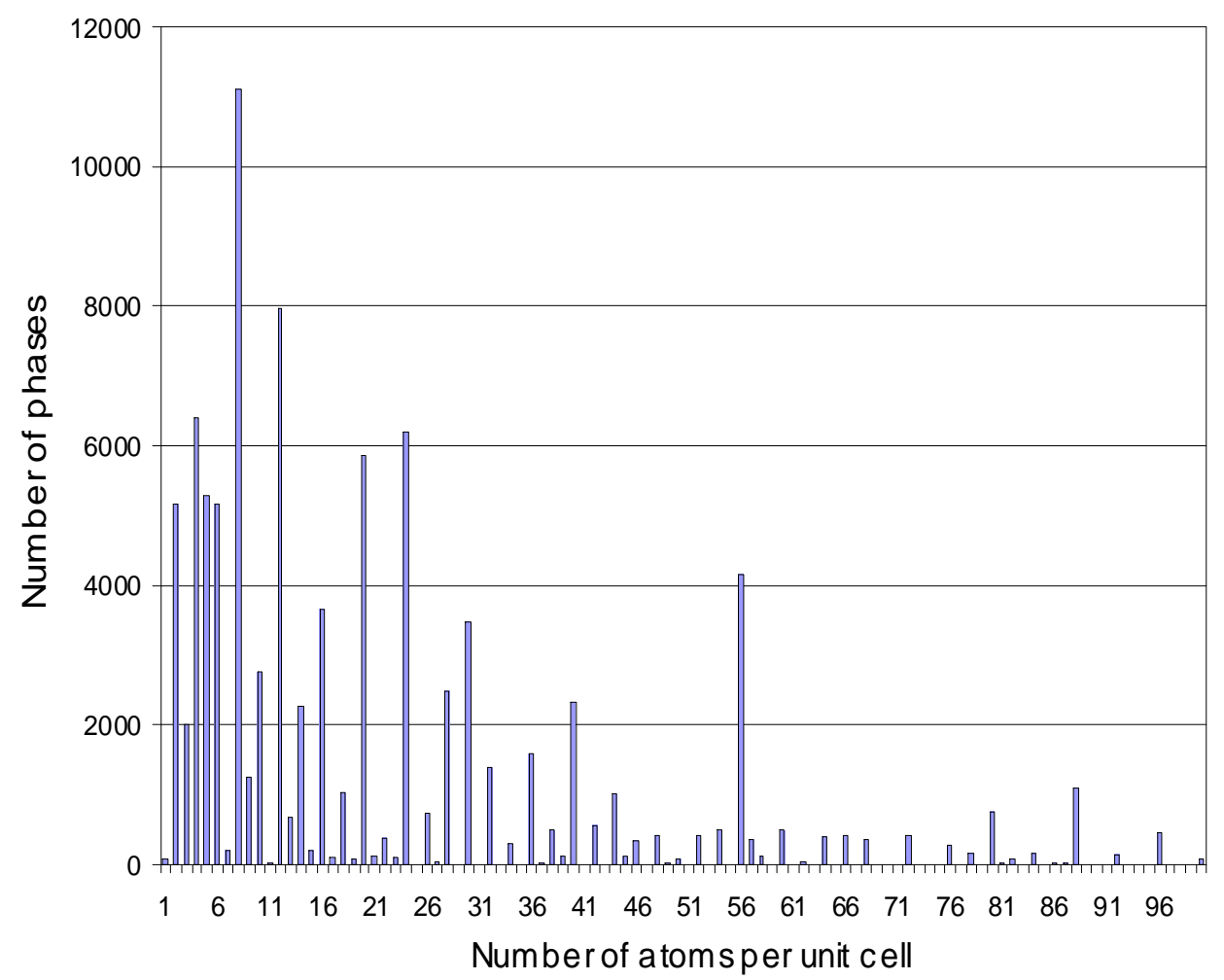

Fig. 10 Number of inorganic phases versus number of atoms per unit cell considering the 1'000 most populous prototypes and their representatives in PCD-2013/14 [6].

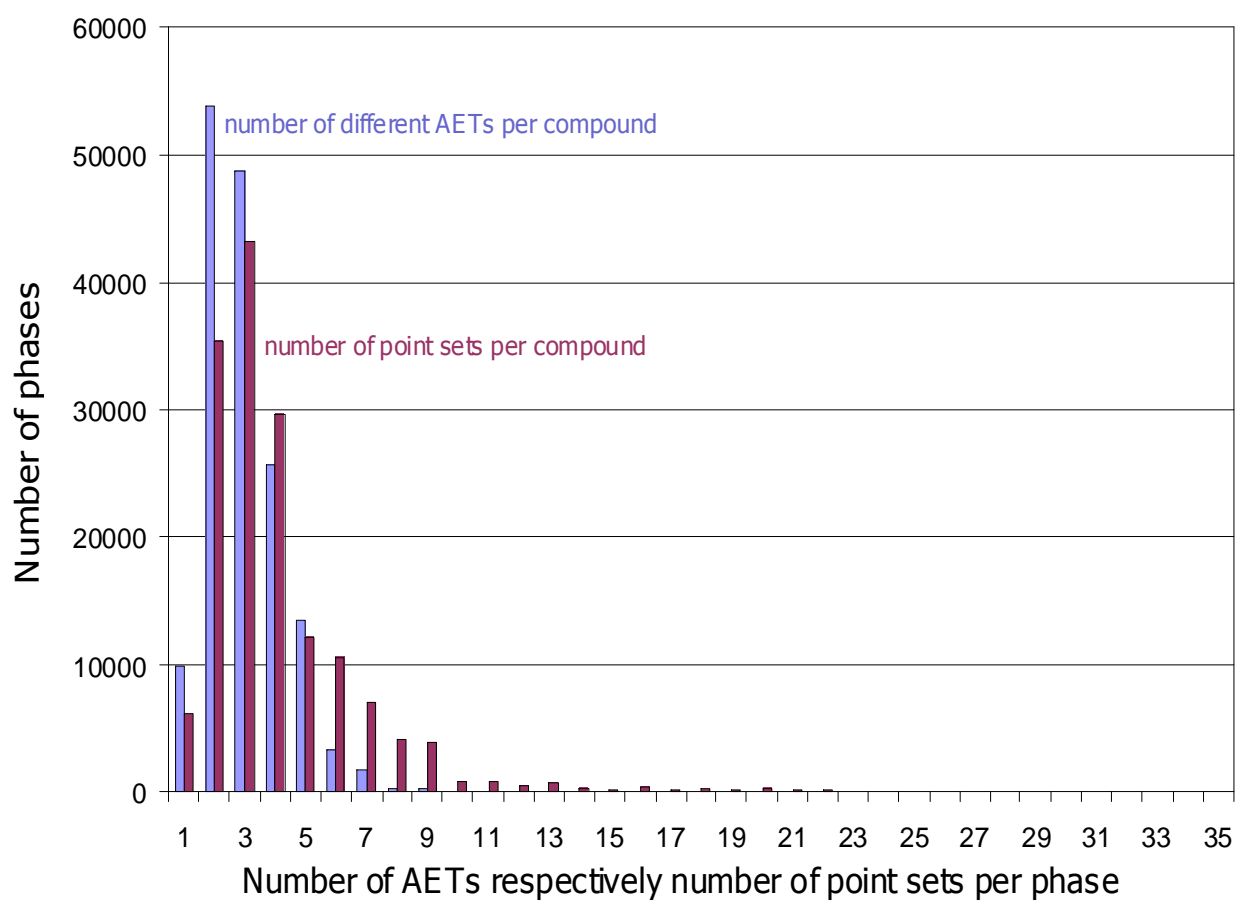

Fig. 11 Number of inorganic phases versus number of different AETs in the structure, respectively number of point sets in the structure, considering the 1'000 most populous prototypes and their representatives in PCD-203/14 [6]. 


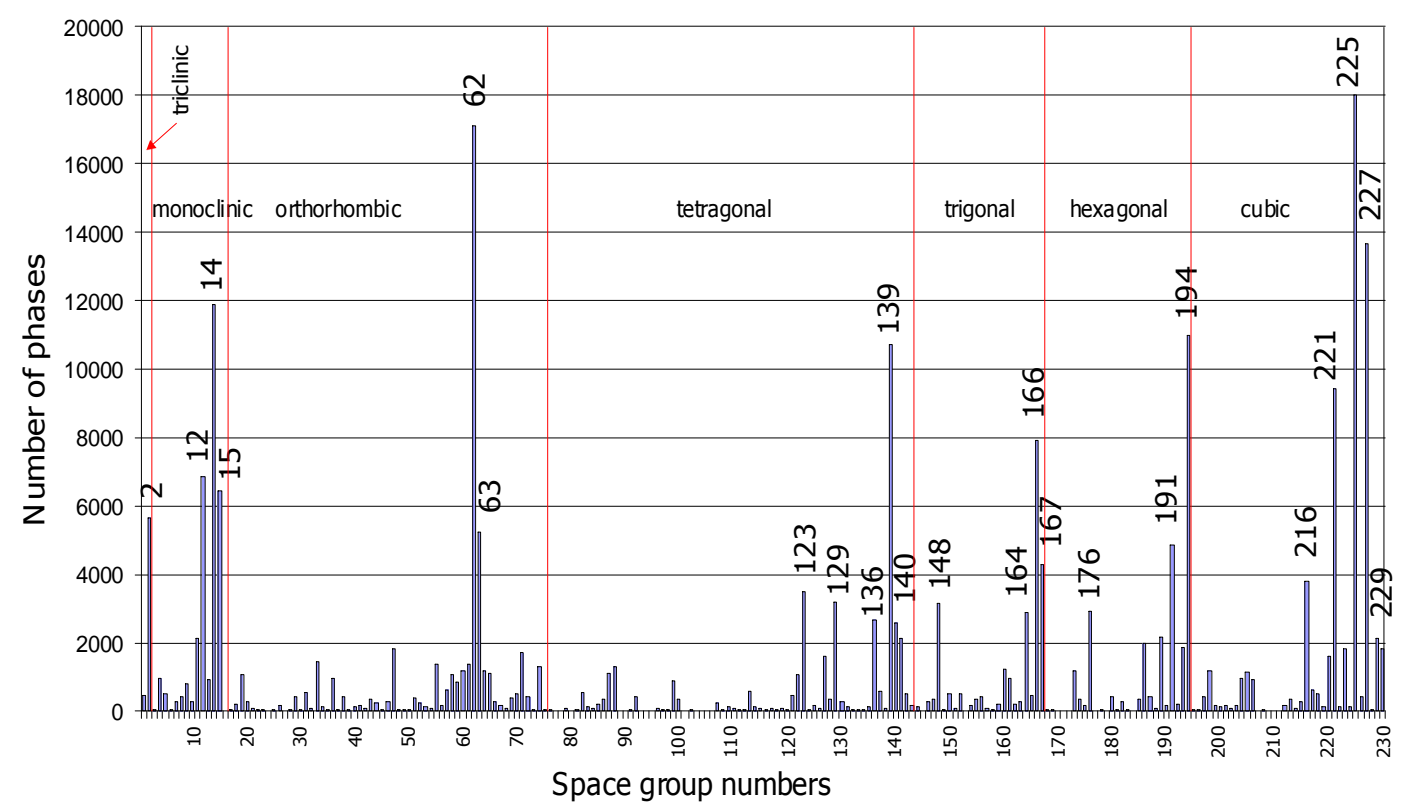

Fig. 12 Number of inorganic phases distributed by space group number considering all entries in PCD-203/14 [6].

inorganics the following space group numbers have to be added: 2 , 14, 15, 123, 129, 136, 140, 148, 164, 167, 176, 229. Within each crystal system a few space groups (the ones with higher symmetry) are preferred. $10 \%$ of the space groups cover $67 \%$ of the inorganic compounds (160'000 of 237'000 compounds).

\section{7) Atomic-environment principle}

Fig. 13 shows a frequency plot for the 18 most populous AETs considering the 1'000 most populous prototypes and their representatives of the PCD-2013/14 [6]. Table 3 lists the 50 most populous AETs among all the refined compounds of PCD- 2013/14 [6].

The atomic-environment principle was formulated in 1994 [17] as follows: The vast majority of all atoms (point sets) in intermetallic compounds have as atomic environment one of 14 polyhedra: tetrahedron, octahedron, cube, tri-capped trigonal prism, fourcapped trigonal prism, icosahedron, cubooctahedron, bi-capped pentagonal pyramid, anti-cubooctahedron, pseudo Frank-Kasper (CN13), 14-vertex FrankKasper, rhombic dodecahedron, 15-vertex FrankKasper, and 16-vertex Frank-Kasper polyhedron. The statement made 20 years ago that 14 AETs are highly preferred is still correct. After having added other classes of inorganic compounds to the intermetallics and alloys, the frequency order has changed (see also Table 3), and the following AETs with low coordination numbers: single atom $(\mathrm{CN}=1)$, collinear $(\mathrm{CN}=2)$, non-linear $(\mathrm{CN}=2)$, collinear triangle $(\mathrm{CN}=3)$, non-coplanar triangle $(\mathrm{CN}=3)$ and square anti-prism $(\mathrm{CN}=8)$ are now among the 14 most populous AETs. In addition it can be stated that 18 out of 100 possible AETs are highly preferred, and were found for $90 \%$ of the point sets considered here (1'002'340 of 1'117'109 point sets). Consequently it appears that Nature strongly prefers certain AETs, most of them being highly symmetrical (except the AETs with $\mathrm{CN}=1$ and $\mathrm{CN}=2$ ).

\section{8) Chemical element ordering principle}

Table 4 gives the number of chemical elements per prototype (rows) versus the number of chemical elements of its representatives (columns), considering the 1'000 most common prototypes and their representatives in PCD-2013/14 [6]. Here we recall what we understand under basic prototype a prototype that has no mixed site occupancy.

Looking at the numbers of Table 4 it is possible to conclude that:

- Compounds with $n$-1 chemical elements cannot be representatives of a basic prototype containing $n$ chemical elements. This is a particular feature of the prototype classification used here, where ordered isopointal structures are distinguished $\left(\mathrm{CaCu}_{5}, \mathrm{hP5}, 191\right.$ and $\mathrm{PrNi}_{2} \mathrm{Al}_{3}, \mathrm{hP5}, 191$ or $\left.\mathrm{CeCo}_{3} \mathrm{~B}_{2}, \mathrm{hP5}, 191\right)$.

- Compounds with $n$ chemical elements that are representatives of a basic prototype containing $n$ chemical elements have no mixed sites (and are therefore called basic representatives).

- Compounds with $n+m$ (where $m$ is an integer $>0$ ) chemical elements that are representatives of a prototype containing $n$ chemical elements have mixed 


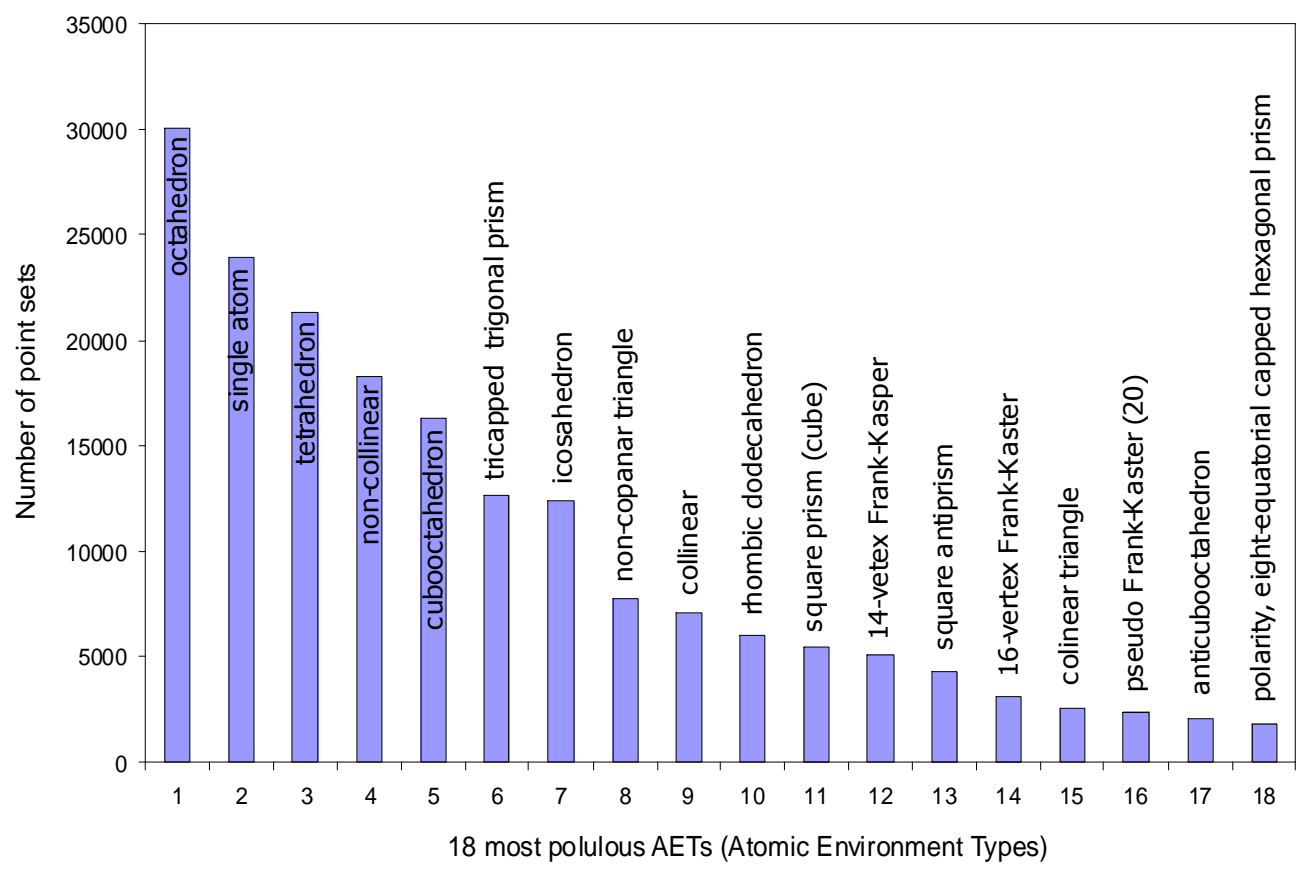

Fig. 13 Total number of point sets for the 18 most frequent AETs considering the 1'000 most populous prototypes and their representatives in PCD-release 2013/14 [6].

sites and generally belong to a solid solution of the basic prototype or one of the basic representatives with $n$ chemical elements.

The 1'000 most populous prototypes and their representatives cover 157'308 entries in PCD-release 2013/14 [6], but represent only 57'518 distinct phases (chemical system + crystal structure). Among the 57'518 distinct phases, only 29'356 have no mixed sites. This means phases (prototypes or representatives) where the number of chemical elements $n$ is the same as for the prototype. The remaining 28'162 phases are representatives of basic prototypes, but have mixed occupation sites, and are in general solid solutions of basic prototypes or their basic representatives. Most of them are therefore not distinct new inorganic compounds. For example, replacement of a few at.\% of the chemical element $A$ in an $A B C$ compound by a closely related chemical element $A^{\prime}$ will lead to a quaternary representative $\left(A, A^{\prime}\right) B C$.

The four principles confirm, with the help of Figs. 10-13 and Table 4, the content of the third cornerstone: "When chemical elements combine to form solids, their crystal structures are beautifully rich, yet very systematic patterns underlie this process." The systematic patterns lead to restraints, nicely reflected in the below listed four principles:

overall simplicity preferred,

high overall symmetry preferred,

high local symmetry reflected through the preference of regular AETs,

high ordering tendency
The combination of the above given experimental facts reduces the number of potential prototypes to be considered in first priority to a few hundred, just $1-2 \%$ of the experimentally known prototypes. The frequency plot in Fig. 14 shows the number of representatives for the 100 most populous prototypes of PCD-2013/14 [6]. The majority of the 32'000 experimentally known prototypes $\left(>25^{\prime} 000\right)$ are unique in the sense that they have no representatives. One of the reasons for the high number of unique prototypes is the significant number of refinements with split sites or other sites very low occupancy, each distinct combination corresponding to a different prototype. For example, almost every refinement of a specific deuteride or hydride phase results in a new prototype, even if the phase is the same.

\subsection{Fourth cornerstone: Laws that link the position of chemical elements within a structure type $\leftrightarrow$ in the Periodic System}

The fourth cornerstone of Nature states that there exist direct links between the position of the constituent chemical element in the Periodic System and its point set occupation within the structure of an inorganic compound.

By definition, inorganic prototypes and their representatives are geometrically very similar to each other. A closer inspection of known inorganic compounds reveals that, for each of the 32'000 prototypes, only a relatively small subset of potential representatives is actually known. The abovementioned link may give us a possibility to predict 


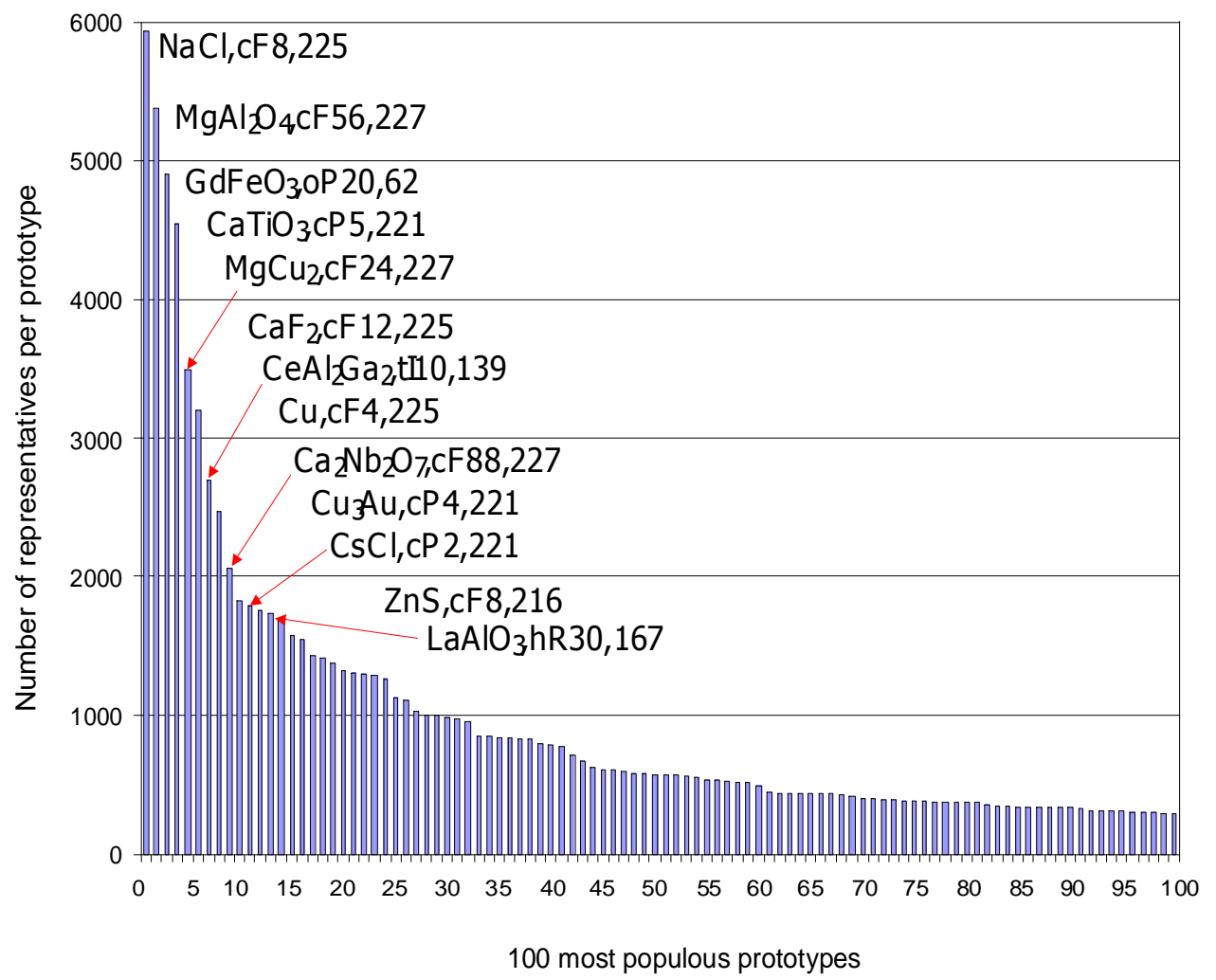

Fig. 14 Number of representatives of each of the 100 most populous prototypes in PCD-release 2013/14 [6].

which compounds will adopt a specific prototype (or a limited selection of prototypes). This is strongly supported by the following four principles:

\section{9) Prototype - Periodic System correlation (chemistry) principle}

This principle was formulated in 1994 [17] as follows: The vast majority of crystal structures show a very strict regularity between the position of the chemical element in the Periodic System ( $s, p, d$, and felements) and its point set(s) occupation within a crystal structure.

By looking at the 1'000 most populous prototypes we can confirm the above statement. About $50 \%$ of the prototypes, in general the ones with few representatives, reveal a simple correlation leading to a few 100 potential combinations. The other 50\%, having in general many known representatives, show a broader correlation, leading to several thousand extrapolated combinations. Fig. 15 demonstrates the chemistry principle with the help of a simple point set - Periodic System correlation for the $\operatorname{ErIr}_{3} \mathrm{~B}_{2}, \mathrm{mS} 12,12$ prototype, where the columns of the Periodic System to which the elements occupying the point sets in the prototype and its representatives belong, have been highlighted. This correlation leads to $6 \times 4 \times(4 \times 2)=144$ element combinations $(1: 2: 3)$, of which 23 have so far been found experimentally. Fig. 16 shows an analog correlation for a more common structure type, $\mathrm{NaFeO}_{2}, \mathrm{hR} 12,166$.
This correlation leads to $(40 \times 103) /(1 \times 2)=2$ '060 combinations (1:1:2). 239 basic inorganic compounds are already known. With the help of these two examples, it is seen that there exists a clear link between the position of the chemical element in the structure and its position in the Periodic System.

10) Structure stability map principle

This principle was formulated in 1994 [17] as follows: The size, electrochemical, valence electron, and atomic-number factors are the factors governing crystal structures of intermetallic compounds. Structure maps separate intermetallic compounds into distinct prototype domains. There exists a whole range of different structure maps for binary and ternary intermetallic compounds. The principle has been further verified and significantly improved since the introduction of the periodic number (PN) in 2008 [13]. The by far simplest and most efficient structure stability maps use PN (and/or expressions of PN) as elemental-property parameter. The best combination was found to be a two-dimensional $\mathrm{PN}_{\max }$ versus mean $\mathrm{PN}_{\min } / \mathrm{PN}_{\max }$ map (for a definition of mean $\mathrm{PN}_{\min } /$ $\mathrm{PN}_{\max }$ see [13]), which has the following five advantages:

i) Inclusion of non-formers, which nicely separate from the formers, is possible.

ii) Applicable to binary, ternary and quaternary systems. 


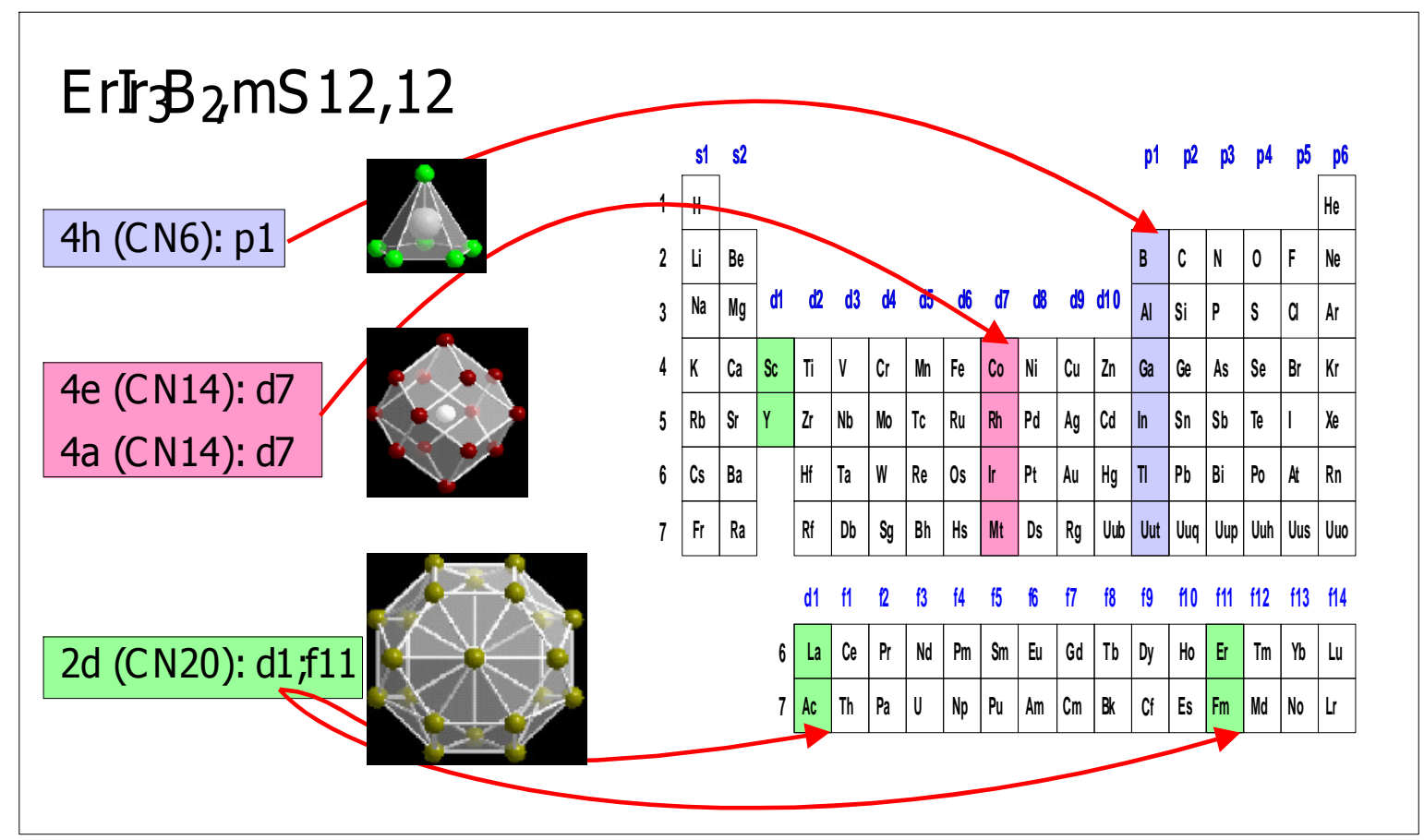

Fig. 15 Chemistry principle shown with the help of a 'simple' point set - spdf-element correlation showing the $\mathrm{ErIr}_{3} \mathrm{~B}_{2}, \mathrm{mS} 12,12$ prototype.

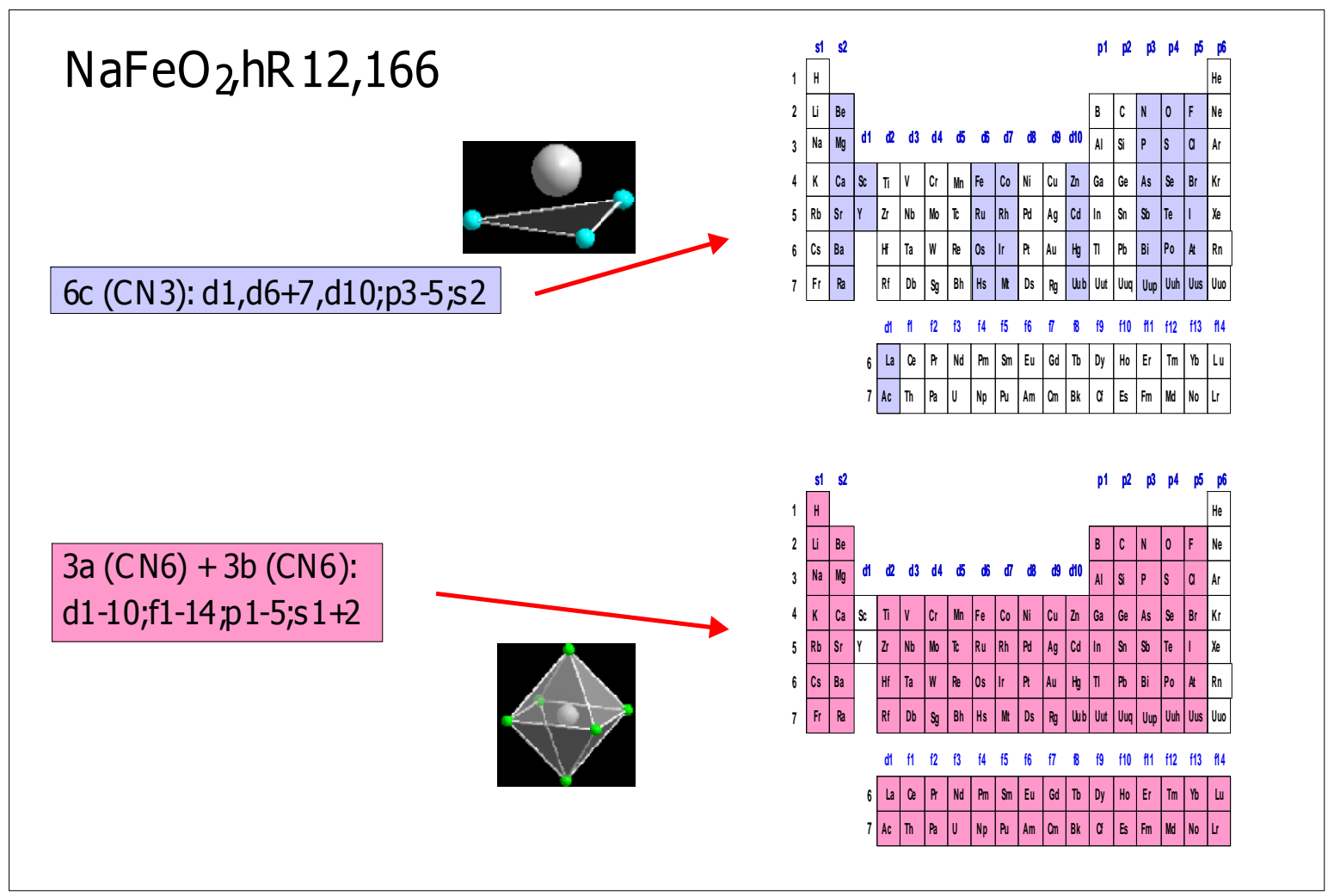

Fig. 16 Chemistry principle shown with the help of a 'complex' point set - spdf-element correlation showing the $\mathrm{NaFeO}_{2}, \mathrm{hR} 12,166$ prototype. 
iii) Iso-stoichiometric prototypes and their representatives can be separated into distinct prototype stability domains in the same map, e.g. binary 1:3 map, ternary 1:1:4 map.

iv) Two-dimensional maps are easy to read and provide obvious prediction ability.

v) $\quad \mathrm{PN}$ is an integer number, known for all chemical elements, and no inaccuracy is coming from the choice of the actual values of the elementalproperty parameter.

This kind of map proved to be very efficient in revealing patterns for different materials problems, e.g. former versus non-former systems; isostoichiometric structure stability maps; or complete solid solubility between binary compounds having the same prototype. It can also separate different atomic environments AETs into distinct stability AET domains in a generalized composition-independent map including binary and multinary compounds [15].

Fig. 17 shows a structure stability map for basic $A B C_{2}$ prototypes and their basic representatives, focusing on the following three prototypes: $\mathrm{NaFeO}_{2}, \mathrm{hR} 12,166$; $\mathrm{Cu}_{2} \mathrm{MnAl}, \mathrm{cF} 16,225$. The majority of the

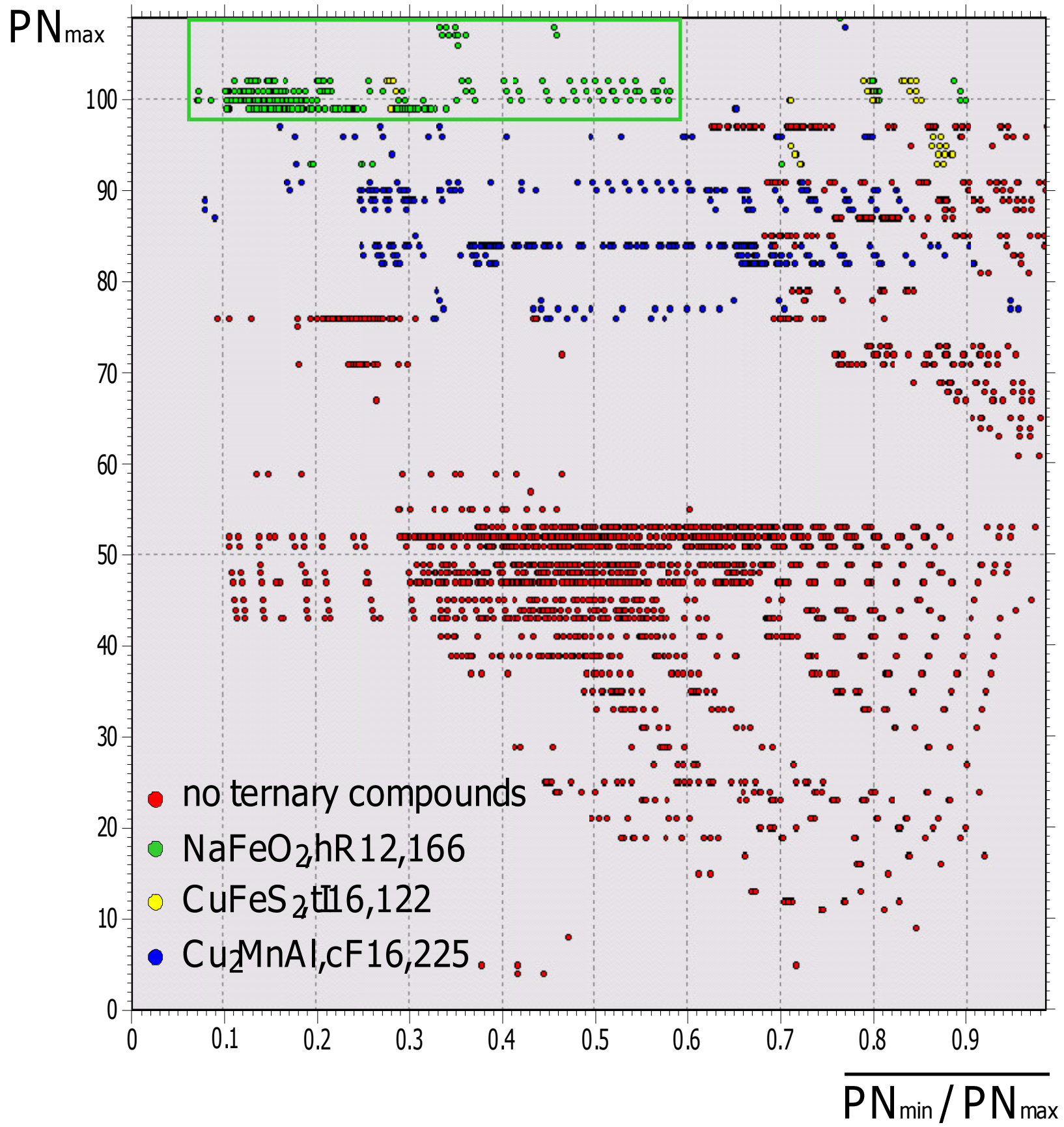

Fig. 17 Structure stability map for $A B C_{2}$ prototypes focusing on the following three prototypes: $\mathrm{NaFeO}_{2}, \mathrm{hR} 12,166$; $\mathrm{CuFeS}_{2}, \mathrm{tI} 16,122 ; \mathrm{Cu}_{2} \mathrm{MnAl}, \mathrm{cF} 16,225$ including all ternary representatives in PCD- 2013/14 [6]. 


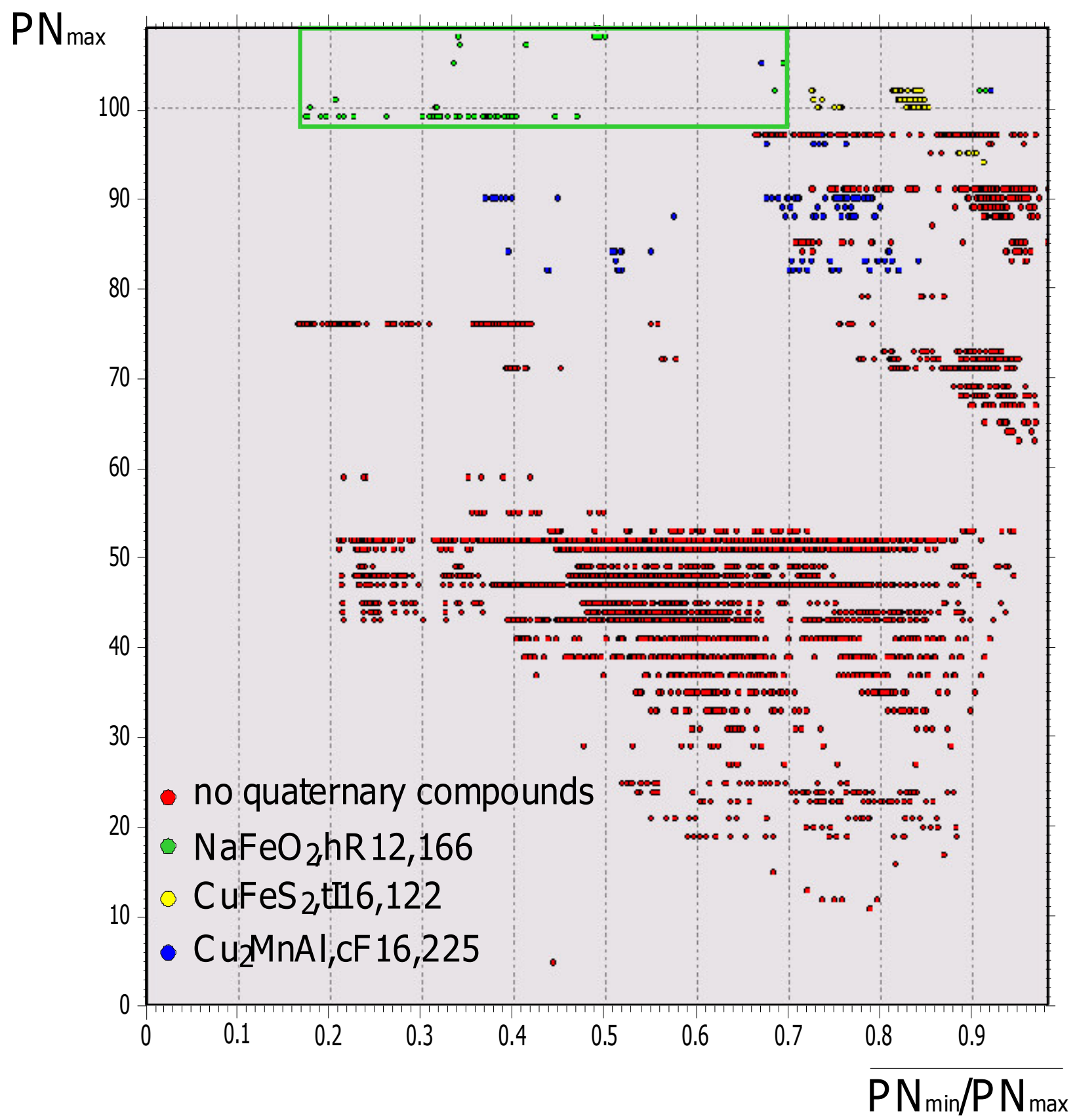

Fig. 18 Structure stability map for $A B C_{2}$ prototypes focusing on the following three prototypes: $\mathrm{NaFeO}_{2}, \mathrm{hR} 12,166 ; \mathrm{CuFeS}_{2}, \mathrm{tI} 16,122 ; \mathrm{Cu}_{2} \mathrm{MnAl}, \mathrm{cF} 16,225$ including all quaternary representatives in PCD- 2013/14 [6].

experimentally known 239 basic ternary representatives of the $\mathrm{NaFeO}_{2}, \mathrm{hR} 12,166$ prototype are nicely located in the domain outlined by a green rectangle. Fig. 18 demonstrates that the same kind of structure stability map can also be used for quaternary 'non-basic' representatives (with mixed site occupancies) of the above-specified basic $A B C_{2}$ prototypes. From the two examples it is seen that there exist clearly defined domains for the representatives of the different prototypes within such iso-stoichiometric structure stability maps. Structure stability maps having as axis expressions of the elemental-property parameter PN provide a direct link to the Periodic System.

\section{1) Generalized AET stability map principle}

This principle was formulated by us in 2008 [15]. Here we give just a short summary: The atomic environment types AETs (coordination polyhedra) observed in binary and multinary inorganic 
compounds were analyzed based on a comprehensive set of literature data. A generalized AET stability map, using as coordinates $\mathrm{PN}_{\max }$ versus $\mathrm{PN}_{\min } / \mathrm{PN}_{\max }$, successfully sub-divided the [central atomcoordinating atoms] combinations where different atomic environment types occur, into distinct 'AET class stability domains'. It was found that chemical elements with $\mathrm{PN}>54$ control the atomic environment types, independently of whether they act as central or as coordinating atoms. The same stability map also showed a clear separation between possible and impossible [central atom-coordinating atoms] combinations. Such stability maps make it possible, for a chemical element $A$ supposed to act as central atom, to predict the AET formed by any coordinating chemical element $B(C, D)$, independently of its concentration and the number of chemical elements involved (see Fig. 19).
The same two-dimensional $\mathrm{PN}_{\max }$ versus (mean) $\mathrm{PN}_{\min } / \mathrm{PN}_{\max }$ coordinates separate, on the one hand different atomic environments AETs into distinct AET stability domains in a generalized compositionindependent structure map including binary and multinary compounds, and on the other hand isostoichiometric prototypes and their representatives into distinct prototype stability domains. This means that these maps can separate element combinations into distinct stability domains, either using the atomic environment type classification (taking each atom as the center) or the prototype classification (which uses as first criterion the Wyckoff sequence). In other words in the first map we focus on the atomic environment of each point set within a prototype and its representatives, in the second map we focus on the crystal structure as an entity. The above said can be seen by comparing Fig. 19 with Fig. 20. Fig. 20 is a

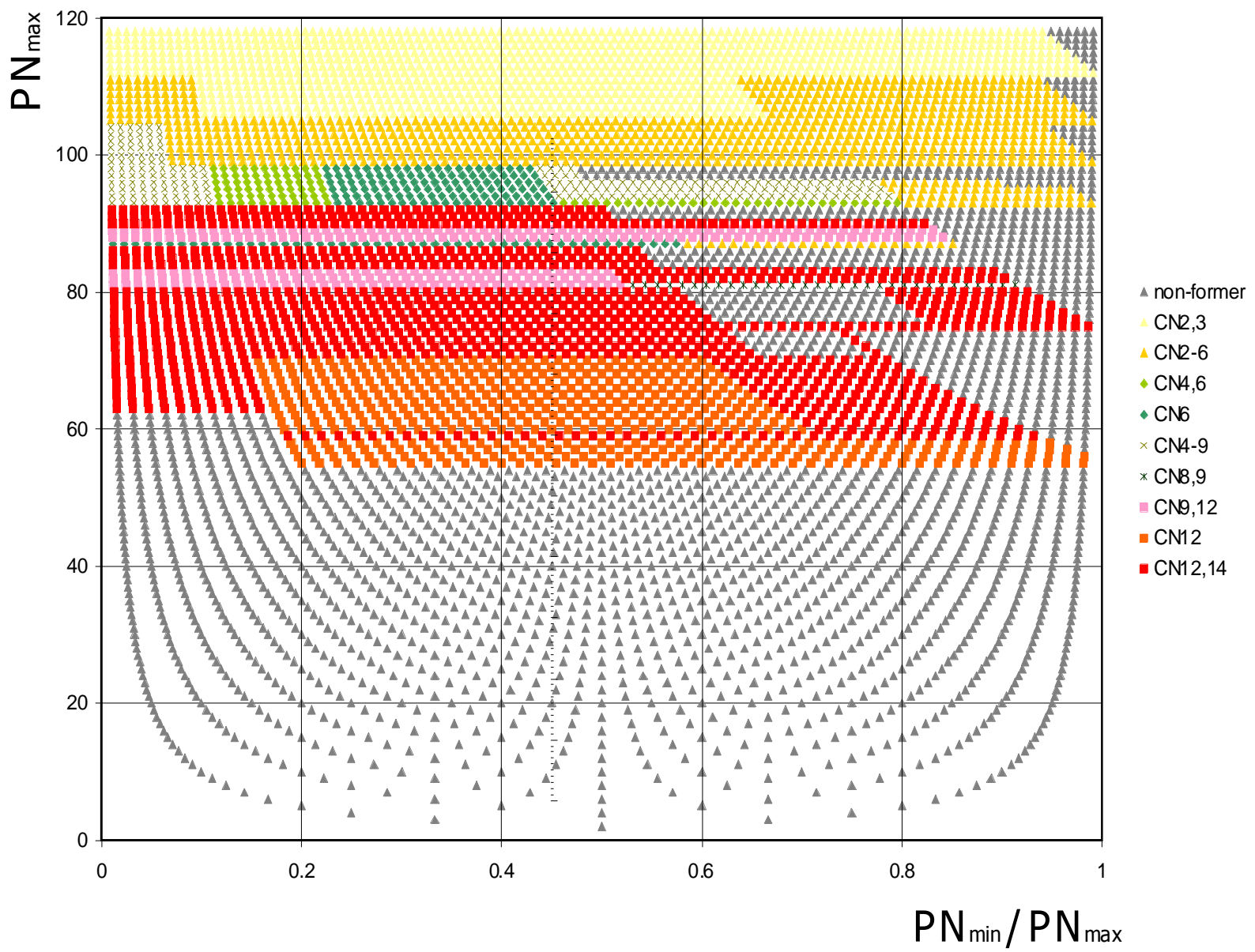

Fig. 19 Generalized AET stability map $\mathrm{PN}_{\max }$ versus $\mathrm{PN}_{\min } / \mathrm{PN}_{\max }$, which is independent of the stoichiometry and the number of chemical elements within an inorganic compound (based on 65'000 inorganic compounds having a [central atom-coordinating atoms]-AET occurrence higher than 3). The map is given for the central atom being $\mathrm{PN}_{\max }$. 
structure stability map for basic quaternary $A B C_{2} D_{4}$ prototypes focusing on the following three prototypes: $\left.\mathrm{H}_{2} \mathrm{~K}_{2} \mathrm{PO}_{4}\right], \mathrm{tI} 24,122$;

$\mathrm{NdBi}_{2} \mathrm{ClO}_{4}, \mathrm{tP} 8,123$;

$\mathrm{Cu}_{2} \mathrm{CdSiS}_{4}, \mathrm{oP} 16,31$ including all quaternary basic representatives. Furthermore, by comparing Figs. 19 and 20 it is seen that basic quaternary prototypes (and representatives) only occur if $\mathrm{PN}_{\max }$ is 90 or larger. This means that at least one of the four constituents has to be one of the following chemical elements: Ge, $\mathrm{Sn}, \mathrm{Pb}, \mathrm{N}, \mathrm{P}, \mathrm{As}, \mathrm{Sb}, \mathrm{Bi}, \mathrm{O}, \mathrm{S}, \mathrm{Se}, \mathrm{Te}, \mathrm{Po}, \mathrm{F}, \mathrm{Cl}, \mathrm{Br}, \mathrm{I}$,
At $\left(p^{2}-p^{5}\right.$ elements). This also supports the conclusion that the diversity of AETs is very much reduced for quaternaries, as compared to binaries and ternaries.

12) Complete solid solution stability map principle This principle was formulated in 1994 [17] as follows: The size, electrochemical, and valence electron factors control solid solubility. Solid-solubility maps were found to separate regions of limited and extended solid solubility for a given chemical element

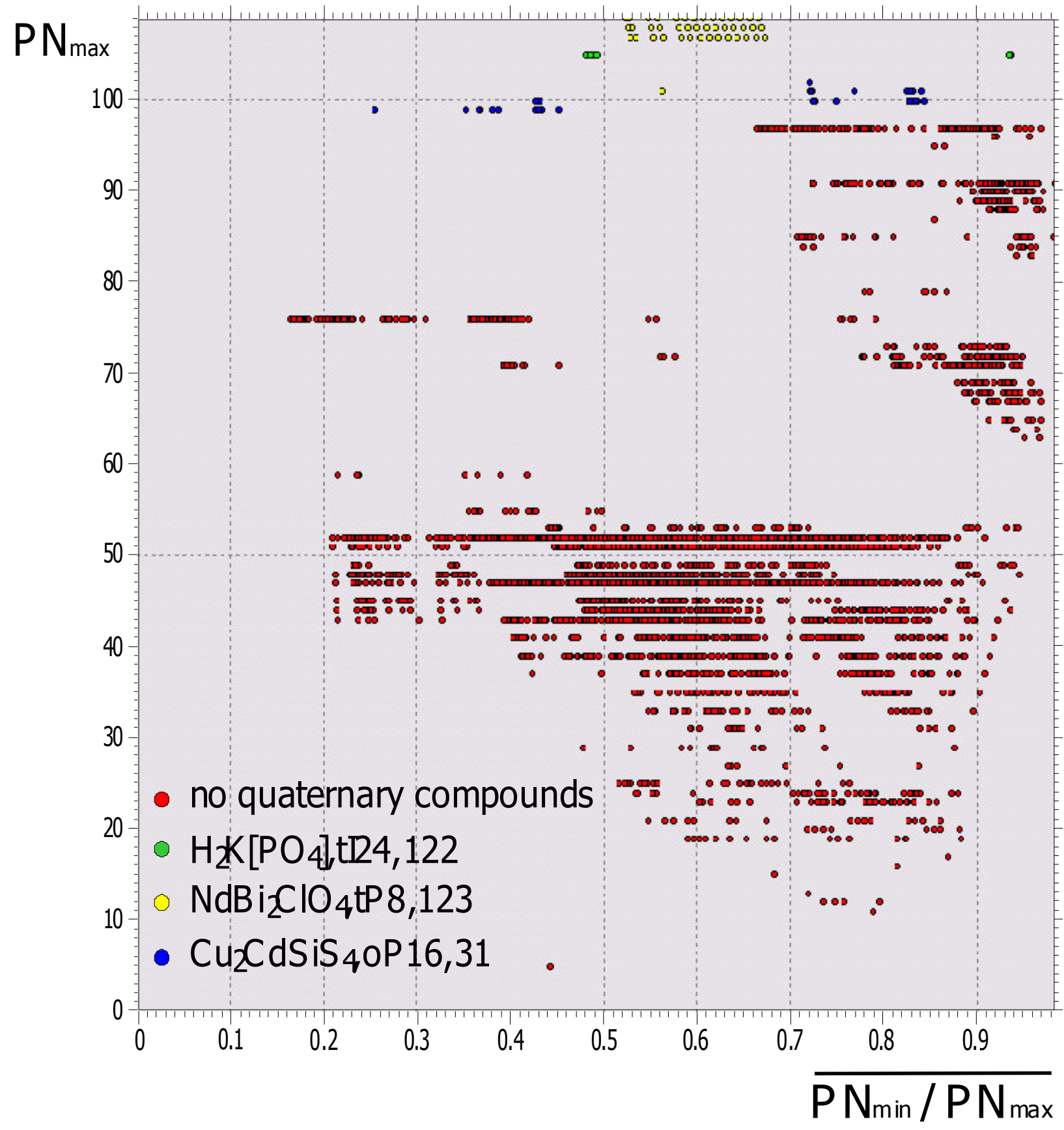

Fig. 20 Structure stability map for quaternary $A B C_{2} D_{4}$ prototypes focusing on the following three prototypes: $\mathrm{H}_{2} \mathrm{~K}_{2}\left[\mathrm{PO}_{4}\right], \mathrm{tI} 24,122 ; \mathrm{NdBi}_{2} \mathrm{ClO}_{4}, \mathrm{tP} 8,123 ; \mathrm{Cu}_{2} \mathrm{CdSiS}_{4}, \mathrm{oP} 16,31$ including all quaternary representatives in PCD-2013/14 [6]. 
solvent. In this work we have extended the study of solid solution behavior from chemical elements to binary compounds as solvents. Since the amount of solid solution data on binaries as solvents is very limited, we focused on a very extreme situation. We searched for ternary systems where the same prototype occurs in two of the binary boundary systems, and either a complete solid solution or limited solid solutions are formed. This requires having access to either appropriate phase diagram data or information about cell parameter(s) versus concentration. We selected the prototype $\mathrm{MgCu}_{2}, \mathrm{cF} 24,227$ which has 238 well established binary representatives. For a 1:2 compound the complete solid solution can be either of the type $A(B, C)_{2}$ or $(B, C) A_{2}$. From the 239 binary compounds one can generate $701 A(B, C)_{2}$ and 1'782
$(B, C) A_{2}$. Considering experimental data showing complete solid solutions, we found $61 A(B, C)_{2}$ and 128 (B,C) $A_{2}$ cases, using data from [6,7].

The prototype $\mathrm{MgCu}_{2}, \mathrm{cF} 24,227$ has two point sets, occupying Wyckoff positions $8 b$ and $16 c$ in the standardized description. The $16 c$ site has as AET an icosahedron with $\mathrm{CN}=12$, and the $8 b$ site a 16 -vertex Frank-Kasper polyhedron with $\mathrm{CN}=16$. Since it is unlikely that the same chemical element will occupy sites with so different AETs, the following six potential solid solutions can be excluded: $\quad A_{2} C \leftrightarrow A B_{2}, \quad A_{2} C \leftrightarrow B C_{2}, \quad A B_{2} \leftrightarrow B C_{2}$, $A_{2} B \leftrightarrow A C_{2}, A C_{2} \leftrightarrow B_{2} \mathrm{C}, \quad B_{2} \mathrm{C} \leftrightarrow A_{2} B$ (see Fig. 21). The $\mathrm{PN}_{\max }$ versus mean $\mathrm{PN}_{\min } / \mathrm{PN}_{\max }$ map in Fig. 22 focuses on the $A(B, C)_{2}$ solid solution case. Here we found experimental data for 42 systems where

B

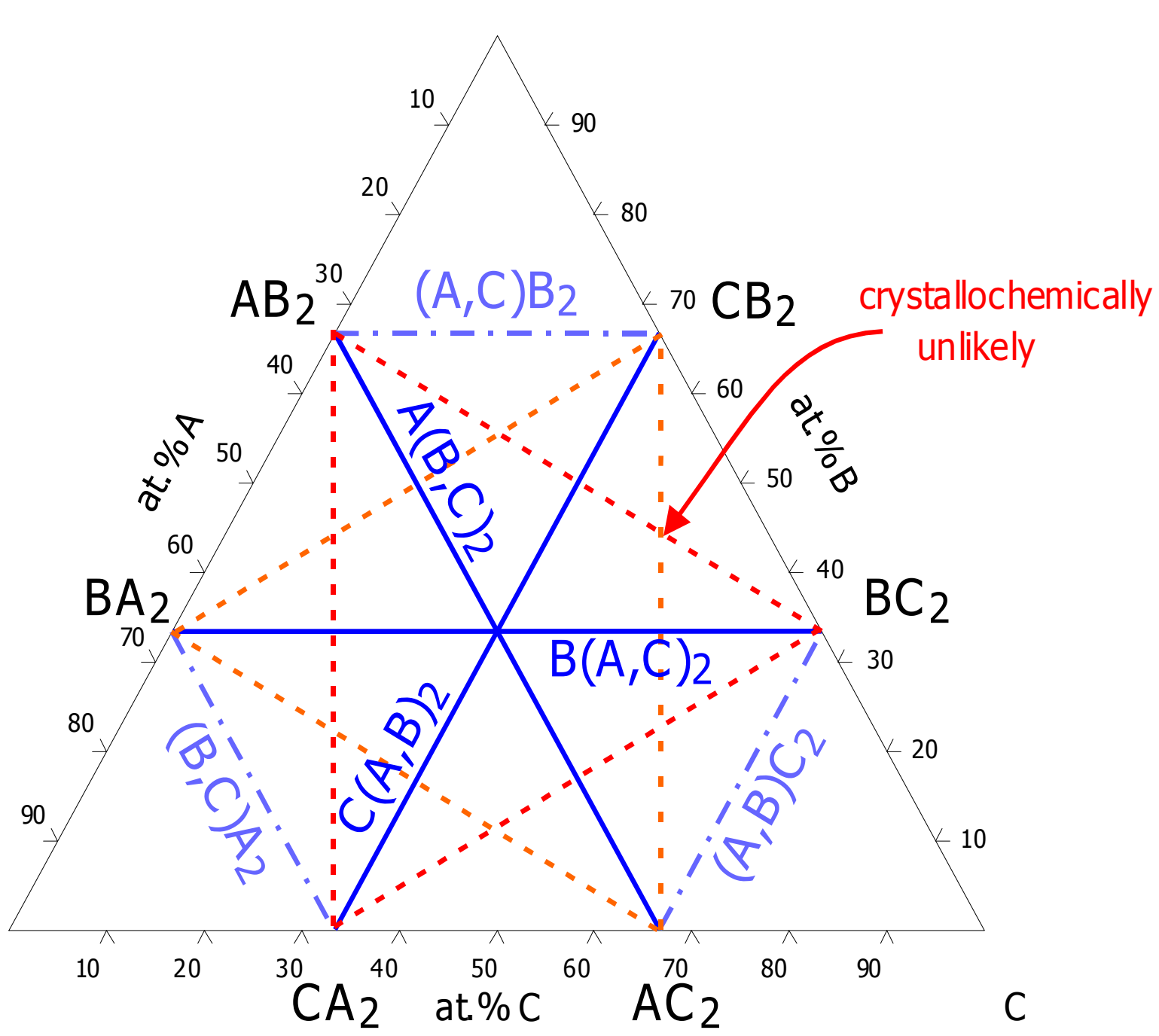

Fig. 21 Possible complete solid solutions between two binaries with the same prototype, shown on the prototype $\mathrm{MgCu}_{2}, \mathrm{cF} 24,227$. 
a complete solid solution does not exist, in addition to the 61 systems where complete solid solution occurs. The stability domain in the $\mathrm{PN}_{\max }$ versus mean $\mathrm{PN}_{\min } / \mathrm{PN}_{\max }$ map for the $\mathrm{MgCu}_{2}, \mathrm{cF} 24,227$ prototype and its representatives is relatively large. Nevertheless the majority of the 61 experimentally confirmed complete solid solutions are located in a relatively small sub-domain (surrounded by a blue line). With the help of these stability domains we can extrapolate: of the 701 possible $A(B, C)_{2}$ combinations, 61 experimental +267 predicted element combinations are of the type 'complete solid solution', while 42 experimental +331 predicted combinations are of the type 'limited solid solution'.

These four principles demonstrate, with the help of Figs. 15-22, the correctness of the fourth cornerstone of Nature: "There exist direct links between the position of the constituent chemical elements in the Periodic System and its point set(s) occupation within the structure of an inorganic compound." The existence of such links is reflected in the above outlined four principles:

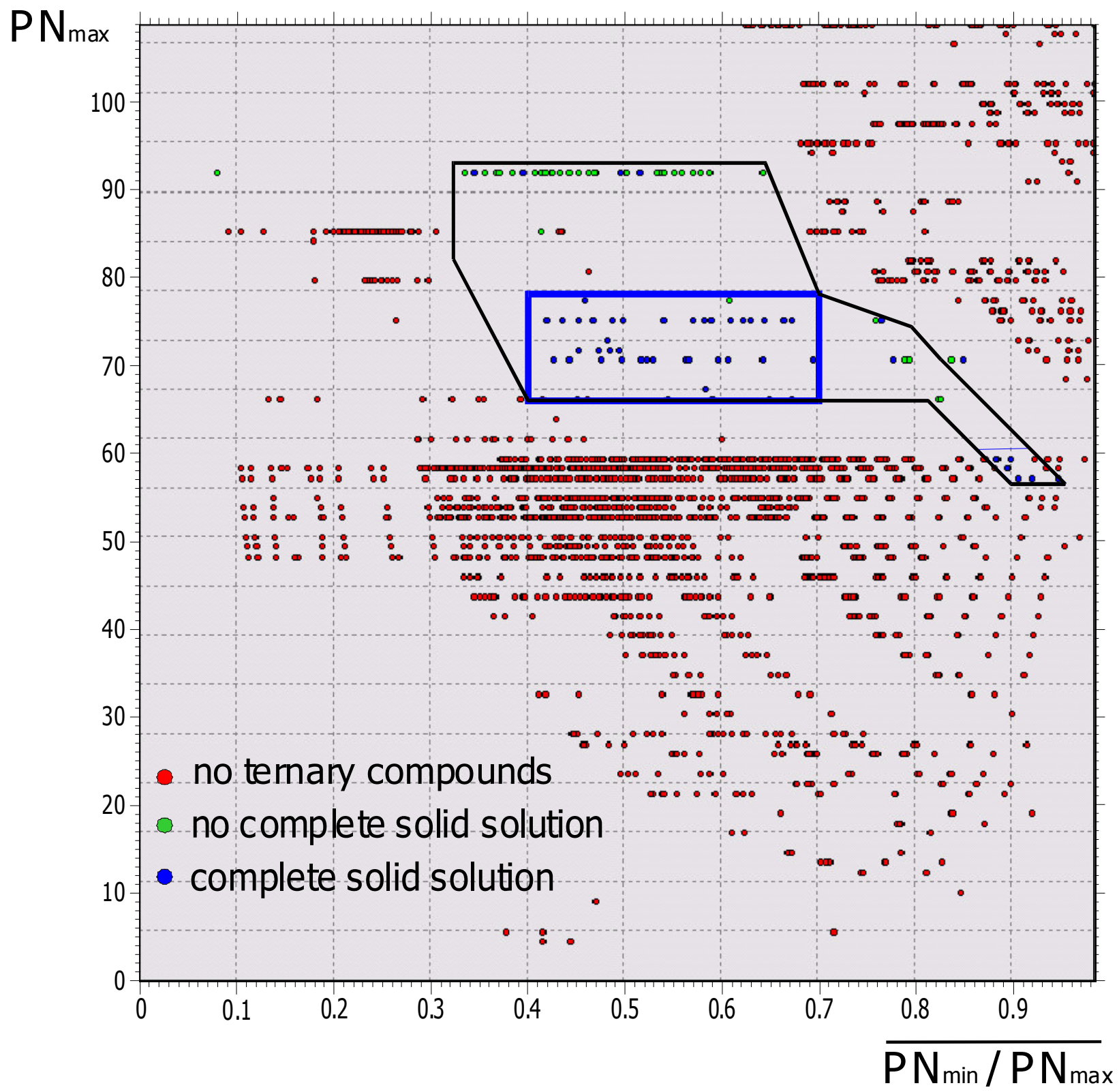

Fig. $22 \mathrm{PN}_{\max }$ versus $\mathrm{PN}_{\min } / \mathrm{PN}_{\max }$ map for complete solid solutions of the type $A(B, C)_{2}$ with the prototype $\mathrm{MgCu}_{2}, \mathrm{cF} 24,227$. 
- Link between the position of the chemical element in the Periodic System and its point set position in the structure (chemistry principle),

- Existence of stability domains for different prototypes and their representatives (structure stability map principle),

Existence of stability domains for different atomic environment types (generalized AET stability map principle),

Existence of solid solution stability domains (solid solution stability map principle).

The above given four principles reduce the number of potential inorganic compounds to be considered from several thousands to a few hundreds for a particular prototype. In addition, it efficiently helps to select from the many potential competing prototypes the most probable ones.

\section{A realistic way to build up a trustworthy calculated part of World Materials}

Based on the 12 principles enumerated above (or the four cornerstones of Nature), we propose a realistic way to build up a trustworthy calculated part of World Materials. There are potentially two major risks to make it untrustworthy. Firstly by calculating potential inorganic compounds belonging to non-former systems, secondly by calculating potential inorganic compounds with improbable prototypes. One should keep in mind that it is still not feasible to calculate all possible 32'000 prototypes, as starting point, for each potential inorganic compound.

We propose the following calculation strategy, which must be dynamically linked to a reference database, such as the PAULING FILE [3-5]:

- Focus on unaries to quaternaries.

- Exclude from all calculations chemical systems clearly belonging to non-formers.

- Focus on known inorganic phases belonging to the most populous prototypes: A) fully refined, B) assigned.

- For each of the most populous prototypes derive point-set $\Leftrightarrow$ periodic system ( $s-, p-, d-, f$-element) correlations (based on known inorganic phases) and select, with the help of structure stability maps (based on known prototypes and atomic environment types), 5-10 competing prototypes: C).

Evaluate for each inorganic phase selected under A)-C) confidence levels by comparing experimental data with own calculated data, as well as the consistency with materials-overview - elementalproperty parameters maps.

Calculate a broad range of physical properties for already known inorganic phases, as well as for predicted phases with a high confidence level of correctness, respectively probability of existence. Evaluate the confidence level for inorganic phases where data have been published.
- Store the calculated data in a fully relational database consistent with the reference database, and create dynamic links so that, in case changes will be done, derived data will be recalculated automatically.

Here we have taken advantage of several statistical plots and materials-overview - elemental-property parameters maps. In addition, the existence of correlations between the positions of the chemical elements in the Periodic System and their positions in the crystal structure, as well as the existence of a fully standardized prototype classification, has been used. Last but not least, the correlation between the number of chemical elements of a compound and the different atomic environment types has proven to be a powerful tool. This all together makes it possible, for each of the most populous prototypes, to populate the atomic sites by chemical elements that are chemically meaningful, and compute structural data and intrinsic physical properties for potentially stable inorganic phases. The resulting set of calculated data will of course include data for the experimentally known inorganic phases contained in the reference PAULING FILE [3-5], which thus serves as validation of the computational approach.

The reliability of the calculated data of World Materials depends on four major factors:

1) Continuous verification of the calculated data by comparison with the reference (experimentally determined data), especially for the structural data, which constitute the starting values for all calculations. This interplay generates a 'structure reliability factor' for calculated structures of inorganic compounds.

2) When the structure has been confirmed, its intrinsic physical properties will be calculated, and again continuously compared with the experimentally known reference data (if available). This interplay generates a 'property reliability factor' for the calculated property data. The same principle should be applied to phase diagram data.

3) Each calculated structure should have an active link to the structure reference data set that was used as starting values. This with the purpose of being able to compare with the experimental values, as well as maintaining the consistency (any change of the experimental reference data will automatically trigger a new calculation procedure).

4) Thorough quality controls of the experimentally obtained data, and data calculated by different methods are required.

\section{Conclusions}

It is known that for $a b$ initio calculations no input other than the laws of quantum mechanics and the atomic numbers (AN) of the involved chemical elements is required. We must then trust that, through the necessary approximations in the calculations applying the laws of quantum mechanics, no relevant 
information goes lost. In principle most of the experimentally determined data contained in the PAULING FILE can be confirmed by calculations, leading to a quantum mechanical materials design approach.

The cornerstones and principles presented in this work are summarized in Table 5. The first cornerstone is a direct consequence of the existence of the about 100 chemical elements given by Nature and their combinations. In practice there exist an infinite number of chemical element combinations (and therefore inorganic compounds). This conclusion is independent of quantum mechanics.

The second to fourth cornerstones of Nature can under no way be derived from quantum mechanics, even each of the about 100 chemical elements possesses all required information, given by Nature, and 'knows what to do' when submitted to external conditions.

Table 5 Summary of cornerstones and principles proposed in this work.

\begin{tabular}{l|l}
\hline $\begin{array}{l}\text { Selection of chemical elements to be combined, } \\
\text { compositions, ...: }\end{array}$ & $\begin{array}{l}\text { Leading to infinitely many } \\
\text { potential inorganic compounds: }\end{array}$ \\
\hline \multicolumn{2}{l}{} \\
\hline First cornerstone of Nature & infinite \\
i) Number of chemical element combinations: & $0-30$ \\
ii) Number of compounds per chemical system: & $>32$ '000 \\
iii) Number of prototypes: &
\end{tabular}

\begin{tabular}{l|l}
\hline Principles (governing factors): & \multicolumn{2}{l}{ Restraints: } \\
\hline \multicolumn{2}{l}{} \\
\hline Second cornerstone of Nature & \multicolumn{2}{l}{ about 30\% } \\
1) Non-formers: & 4 '087'975 systems \\
2) No. chemical elements per compound - AET correlation: & $10 \%$ (2 elements); 15\% (3); 38.6\% (4) \\
3) Inactive composition range: & 10 (2 elements); 769 (3) \\
4) Most probable stoichiometry ratios: &
\end{tabular}

\begin{tabular}{l|l}
\hline Third cornerstone of Nature & \\
5) Simplicity (90\% probability): & $\begin{array}{l}<40 \text { atoms/unit cell } \\
<10 \text { point sets } \\
\end{array}$ \\
& $<5$ AET \\
23 of 230 space groups \\
6) Symmetry (90\% probability): & 18 of 100 AETs \\
7) Atomic environment type (90\% probability): & $(n-1)$ elements impossible \\
$8)$ Ordering tendency: & \\
\hline
\end{tabular}

\begin{tabular}{l|l}
\hline Fourth cornerstone of Nature & \\
9) Prototype - periodic system correlation: & simple to broad \\
10) Structure stability maps: & stability domains \\
11) Generalized AET stability map: & stability domains \\
12) Solid solution stability map: & stability domain \\
\hline
\end{tabular}


The cornerstones can only be discovered by the examination of a large amount of critically evaluated experimentally determined data. Since we do not have the ability to cross-link all the consequences of the interactions of the atoms under all possible conditions (e.g. nature of the chemical elements to be combined, stoichiometric ratios, temperature, pressure, etc.), we have no other choice than to focus on different consequences of the above mentioned causes. The second cornerstone focuses on the fundamental ability to form an inorganic compound. The third cornerstone focuses on the ordering of the atoms within an inorganic compound. And, finally, the fourth cornerstone reveals the link between the position of a chemical element in the Periodic System and its position(s) in the crystal structure of a particular inorganic compound. These four cornerstones can be considered as different ways to look at the same cause. They are supported by the twelve principles outlined in Table 5. These ultimately lead to restraints, which are a requirement for the development of a practicable and trustworthy materials design approach.

The power of critically evaluated data is considerable, knowing that already 20 years ago, having access to only $20 \%$ of the now available data, it was possible to derive the core content for nine principles correctly. Apparently the amount of data with information on about 30'000 phases was already sufficient to derive reliable materials knowledge. The in this work verified, and where necessary, extended, nine principles, as well as the newly discovered three principles are based on a much more robust database system with information on over 140'000 inorganic phases. We thus conclude that the here-formulated twelve principles, and from them derived restraints, must be considered trustworthy.

\section{Acknowledgements}

We would like to thank Dr. Erich Wimmer and Dr. Paul Saxe of Materials Design Inc. for interesting and stimulating discussions in context with the World Materials calculated part.

\section{References}

[1] J. Gray, The Fourth Paradigm, Data-Intensive Scientific Discovery, In: T. Hey, S. Tansley, K. Tolle (Eds.), Microsoft Corp., Redmond, Washington, USA, 2009 pp. xvii-xxxi.

[2] J.P. Holdren, Materials Genome Initiative for Global Competitiveness 1-18, Executive Office of the President of the United States, National Science and Technology Council, 2011.
[3] http://www.paulingfile.com/

[4] P. Villars, K. Cenzual, J.L.C. Daams, F. Hulliger, T.B. Massalski, H. Okamoto, K. Osaki, A. Prince (Eds.) PAULING FILE Binaries Edition, on CD-ROM, ASM International, Materials Park, Ohio, USA, 2002.

[5] http://crystdb.nims.go.jp/index_en.html

[6] P. Villars, K. Cenzual (Eds.), Pearson's Crystal Data: Crystal Structure Database for Inorganic Compounds, on CD-ROM, Release 2013/14, ASM International, Materials Park, Ohio, USA, 2013.

[7] P. Villars, H. Okamoto, K. Cenzual (Eds.), ASM Alloy Phase Diagram Database; http://www.asminternational.org/AsmEnterprise/ APD, ASM International, Materials Park, Ohio, U.S.A., 2013/14.

[8] $P D F-4^{+}$, on CD-ROM, Release 2013/14, International Centre for Diffraction Data (ICDD), Newtown Square, Pennsylvania, U.S.A.

[9] P. Villars, K. Cenzual, H. Okamoto, F. Hulliger (Eds.), Springer Materials, The LandoltBörnstein Database: Inorganic Solid Phases, 2013.

[10] K. Cenzual, M. Berndt, K. Brandenburg, V. Luong, E. Flack, P. Villars, ESDD software package, copyright Japan Science and Technology Corporation, 2000; updates by O. Shcherban, SCC "Structure Properties", Lviv, Ukraine.

[11] E. Parthé, L. Gelato, B. Chabot, M. Penzo, K. Cenzual, R. Gladyshevskii, Gmelin Handbook of Inorganic and Organometallic Chemistry, $8^{\text {th }}$ Ed., TYPIX - Standardized Data and Crystal Chemical Characterization of Inorganic Structure Types, 4 vols., Springer, Heidelberg, 1993-1994.

[12] L.M. Gelato, E. Parthé, J. Appl. Crystallogr. 20 (1987) 139-143.

[13] P. Villars, J. Daams, Y. Shikata, K. Rajan, S. Iwata, Chem. Met. Alloys 1 (2008) 1-23.

[14] P. Villars, K. Cenzual, J. Daams, Y. Chen, S. Iwata, J. Alloys Compd. 317-318 (2004) $167-175$.

[15] P. Villars, J. Daams, Y. Shikata, Y. Chen, S. Iwata, Chem. Met. Alloys 1 (2008) 210-226.

[16] P. Villars, Y. Chen, S. Iwata, Sci. Council Jpn. J., 2014, accepted.

[17] P. Villars, In: J.H. Westbrook, R.L. Fleischer (Eds.), Intermetallic Compounds, Principles and Practice, Wiley, New York, 1994, Vol. 1, pp. 227-275.

[18] P. Villars, L.D. Calvert, Pearson's Handbook of Crystallographic Data for Intermetallic Phases, Second Edition, 4 vols., ASM International, Materials Park, Ohio, USA, 1991.

[19] ICSD, FIZ/NIST, Fachinformations-Zentrum Karlsruhe, Germany, 2013. 
P. Villars, S. Iwata, PAULING FILE verifies / reveals 12 principles in materials science ...

[20] F.R. de Boer, W.C.M. Mattens, A.R. Miedema, A.K. Niessen, In F.R. de Boer, D.G. Pettifor (Eds.), Cohesion in Metals, Transition Metal Alloys (Cohesion and Structure), North-Holland, Amsterdam, Netherlands, Vol. 1, 120 p.

[21] P. Villars, J. Less-Common Met. 119 (1986) $175-188$.
[22] J.L.C. Daams, In: J.H. Westbrook, R.L. Fleischer (Eds.), Intermetallic Compounds, Principles and Practice, Wiley, New York, 1994, Vol. 1, pp. 363-383.

[23] P.I. Kripyakevich, Structure Types of Intermetallic Compounds, Nauka, Moscow, 1977, 288 p. (in Russian). 